hep-th/0608217

NSF-KITP-06-26

CERN-PH-TH/2006-151

\title{
Mergers and Typical Black Hole Microstates
}

\author{
Iosif Bena ${ }^{(1)}$, Chih-Wei Wang ${ }^{(2)}$ and Nicholas P. Warner ${ }^{(2,3)}$ \\ (1) School of Natural Sciences, Institute for Advanced Study \\ Einstein Dr., Princeton, NJ 08540, USA \\ (2) Department of Physics and Astronomy, University of Southern California \\ Los Angeles, CA 90089-0484, USA \\ (3) Department of Physics, Theory Division \\ CERN, Geneva, Switzerland
}

\begin{abstract}
We use mergers of microstates to obtain the first smooth horizonless microstate solutions corresponding to a BPS three-charge black hole with a classically large horizon area. These microstates have very long throats, that become infinite in the classical limit; nevertheless, their curvature is everywhere small. Having a classically-infinite throat makes these microstates very similar to the typical microstates of this black hole. A rough CFT analysis confirms this intuition, and indicates a possible class of dual CFT microstates.

We also analyze the properties and the merging of microstates corresponding to zeroentropy BPS black holes and black rings. We find that these solutions have the same size as the horizon size of their classical counterparts, and we examine the changes of internal structure of these microstates during mergers.
\end{abstract}

August 2006 


\section{Introduction}

In [1] we found a very large number of geometries that have exactly the same supersymmetries, size, charges and angular momenta as maximally-rotating, three-charge BPS black holes and black rings in five dimensions. These smooth, horizonless solutions have no brane sources, and belong to the class constructed and analyzed in [2,3], based on earlier work in [4].

These solutions are very similar to BPS black holes and BPS black rings [8, 9, 6, 10, 17] and only differ from those near the would-be "classical" horizon. In the new, horizonless solutions this region is smooth and compact, and contains a large number of topologically non-trivial two-cycles. Hence these solutions are called "bubbled black holes" and "bubbled black rings." All these solutions can be dualized to a frame in which they are asymptotic to $A d S_{3} \times S^{3} \times T^{4}$, and therefore are dual to states in the D1-D5-P CFT that describes black holes and black rings. Understanding whether this kind of supergravity solutions are dual to typical CFT states is perhaps among the most important questions in understanding black holes in string theoryl 1 .

For practical reasons, all the five-dimensional, three-charge BPS microstates that have been constructed [13-17, 1, 2, 3] use as a base a four-dimensional "generalized hyper-Kähler" metrid 2 that has a tri-holomorphic $U(1)$ symmetry, and is thus a Gibbons-Hawking (GH) metric. Nevertheless, as recently shown in [1], using a Gibbons-Hawking base generically appears to yield bubbled solutions corresponding to black rings and black holes of zero horizon area. Indeed, it appears that none of the microstate solutions currently in the literature has the same charges and angular momenta as a black hole with a classically large horizon area.

It is very important to understand whether this limitation arises from using a GibbonsHawking base. If this were so, then we would need to find more general classes of hyperKähler base metrics, and these are very hard to construct and analyze explicitly. Before embarking on this difficult task, one should therefore first attempt to construct microstates

1 If the answer to this question is positive, then the $A d S$-CFT correspondence would force us to think about black holes as ensembles of smooth horizonless geometries, which would greatly deepen our understanding of black holes, and quantum gravity in general. A review of this can be found in [11] and [12.

2 By "generalized," we mean a hyper-Kähler base whose metric is allowed to change its overall sign in compact regions, thereby flipping the signature from +4 to -4 . 
of positive-entropy black holes starting from a Gibbons-Hawking base. Again this is primarily because of computational convenience: Such solutions would be much easier to obtain explicitly, analyze, and relate to the corresponding CFT microstates.

An obvious place to start would be to examine the merger of two black holes, which is always irreversible. Nevertheless, such a configuration can preserve at most and $S O(3)$ that has no tri-holomorphic $U(1)$ subgroups; therefore the merger of two black hole microstates cannot be described using a GH base metric. On the other hand, the merger of a black hole with a black ring in its equatorial plane preserves a $U(1) \times U(1)$ symmetry. Hence, using a result of [18], we expect the merger of the corresponding microstates to be described using a GH base metric.

The merger of a BPS black hole and a BPS black ring can be reversible or irreversible, depending on the charges of the two objects [19]. One therefore expects the merger of microstates to result in an zero-entropy $\mathrm{BH}$ microstate or an positive-entropy $\mathrm{BH}$ microstate3, depending on the charges of the merging microstates. Moreover, since the merger can be achieved by keeping the base Gibbons-Hawking, one expects the resulting positive-entropy BH microstate to have a Gibbons-Hawking base.

The main purpose of this paper is to study the merger of zero-entropy BR microstates and zero-entropy $\mathrm{BH}$ microstates. We construct solutions that describe a large, bubbled black ring with a bubbling black hole in the center, and reduce some flux parameters in order to bring them together. We find that, when seen from far away, the merger of microstates closely parallels the merger of the corresponding classical counterparts. In particular, the merger happens at the same values of the charges and angular momenta, and the resulting microstate is always that of a BMPV black hole.

On the other hand, we find that there is a huge qualitative difference between the behavior of the internal structure of microstates in "reversible" and "irreversible" mergers]. A "reversible" merger of an zero-entropy BH microstate and an zero-entropy BR microstate produces another zero-entropy $\mathrm{BH}$ microstate. We find that the bubbles corresponding to

3 Obviously, the compound adjectives, "positive-entropy" and "zero-entropy" are intended to be applied to the black hole and black ring whose microstate geometries we discuss, and not to the horizonless microstate geometries themselves.

4 With an obvious abuse of terminology, we will refer to such solutions as "reversible" and "irreversible" mergers of microstates with the understanding that the notion of reversibility refers to the classical $\mathrm{BH}$ and $\mathrm{BR}$ solutions to which the microstates correspond. 
the ring simply join the bubbles corresponding to the black hole, and form a bigger bubbled structure.

In an "irreversible" merger, as the ring bubbles and the black hole bubbles get closer and closer, we find that the distances between the GH points that form the black hole foam and the black ring foam also decrease. As one approaches the merger point, all the sizes in the GH base scale down to zero while preserving their relative proportions. In the limit in which the merger occurs, the solutions have $J_{1}=J_{2}<\sqrt{Q_{1} Q_{2} Q_{3}}$, and all the points have scaled down to zero size on the base. Therefore, it naively looks like the configuration is singular; however, the full physical size of the bubbles also depends on the warp factors, and taking these into account one can show that the physical size of all the bubbles in the black hole and black ring foams remains the same. The fact that the GH points get closer and closer together implies that the throat of the solution becomes deeper and deeper, and more and more similar to the throat of a BPS black hole (which is infinite).
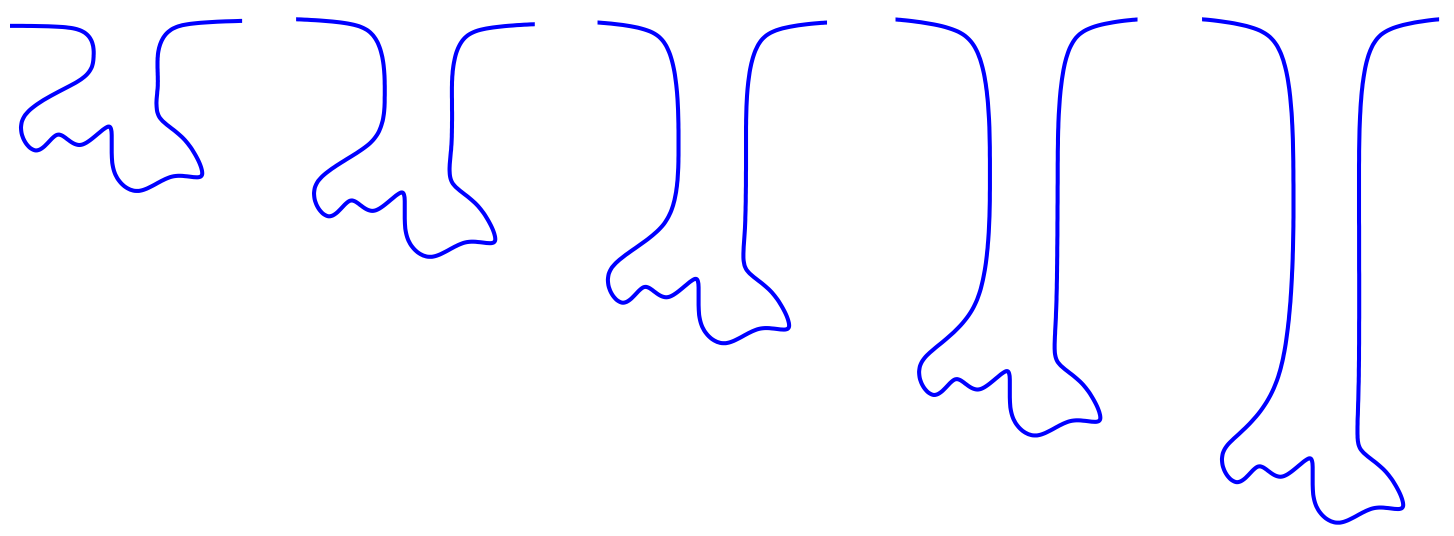

Fig. 1: A heuristic depiction of an irreversible microstate merger. Throughout the merger the physical size of the bubbles remains fixed, but the throat of the solutions deepens.

Our investigation therefore shows that Gibbons-Hawking-base microstates of positiveentropy black holes can be obtained from mergers. Moreover, these microstates appear to want to have very long throats, and consequently very high red-shifts. As we will see, the quantization of the fluxes does not allow the scaling to continue ad infinitum; the length of the throats of microstate solutions only becomes infinite in the classical limit.

Hence, at least for a Gibbons-Hawking base, the crucial difference between zeroentropy $\mathrm{BH}$ microstates and positive-entropy $\mathrm{BH}$ microstates obtained from mergers is the length of the throat. The throat of the zero-entropy $\mathrm{BH}$ microstates remains finite 
in the classical limit, while the throat of the positive-entropy $\mathrm{BH}$ microstates becomes infinite. In the rest of this paper we will refer to the microstates of positive-entropy BH's as "deep" microstates5, and to the zero-entropy BH microstates as "shallow" microstates.

As we have mentioned above, all the solutions we construct are dual to states of the D1-D5-P CFT. A heuristic way to describe the states of this system is to consider a long effective string of total length $N_{1} N_{5}$ that is broken into component strings, on which one puts $N_{P}$ units of momentum. The CFT entropy comes from the various ways of distributing the momentum between the different modes of the component strings. The typical CFT states, dual to the black hole, have only one component string, that carries all the momentum; atypical states have more component strings [20,21]. The energy gap in the CFT is the inverse of the length of the largest component string. One can also compute the mass gap in the bubbling solutions, as a function of the charges and angular momentum, and compare it to the gap computed in the CFT. A heuristic analysis then indicates that the deep microstates with left-moving angular momentum $J_{L}$ are dual to states with $J_{L}$ component strings of length $N_{1} N_{5} / J_{L}$. The deepest of the deep microstates have $J_{L}=1$, and therefore appear to be dual to CFT states with a single component string. While this is not enough to establish that deep microstates are typical - after all, they might be dual to an atypical distribution of momentum on the component string the fact that they are dual to CFT states with one component string makes them very similar to typical microstates.

In addition to obtaining microstates corresponding to black holes with classically large entropy, we can use mergers to investigate the "size" of microstates. When a classical black hole and a classical black ring merge, their horizons touch. Our results indicate that in a similar fashion, when a bubbled black hole and a bubbled black ring merge, their bubbles get very close to each other. This shows that the bubbling black hole microstates constructed in [1] have the same size and macroscopic features as the corresponding classical black hole.

Another purpose of this paper is to discuss, in more detail, the construction of bubbled black holes and black rings outlined in [1], and to analyze more thoroughly the entropy of the bubbled black holes and black rings with a GH space. Our analysis of mergers can also be used to find the detailed features of the distribution of the GH points that form bubbled

5 We originally considered calling these microstates "deep-throat microstates" but decided against it to avoid the obvious tasteless jokes. 
black holes and bubbled black rings, as well as the behavior of the warp factors inside these solutions, and is important for obtaining a deeper understanding of their physics.

In section 2 we summarize the structure and properties of the five dimensional smooth horizonless solutions of [2, 3]. In section 3 we give an expanded version of the construction of bubbling black holes and black rings in [1]. We also give some numerical results that suggest how the GH centers arrange themselves within the bubbling solutions. In section 4 we analyze the merger of microstates analytically, and show that one can rederive the merger conditions found in [19] for classical rings and holes, using the bubbled geometries. In section 5 we analyze analytically and numerically the irreversible mergers, which result in positive-entropy BH microstates. These, to our knowledge, are the first smooth horizonless solutions that have the same charges and angular momenta as a black hole with a classically large horizon area. In section 6 we look at some examples of reversible mergers and show that during such mergers the separation of the ring and the black hole bubbles becomes very small, resulting in a configuration where the two bubbling structures essentially touch. In section 7 we make a heuristic $A d S$-CFT analysis of the deep microstates, and argue that the deepest microstates correspond to CFT states that have a single component string; these microstates are therefore in the same class as the typical microstates of this black hole. Section 8 contains conclusions and suggestions for future research.

\section{A review of the bubbling solutions}

\subsection{The solution}

The full eleven-dimensional metric of a BPS black-hole and black-ring solutions has the form:

$$
\begin{aligned}
d s_{11}^{2} & =-\left(\frac{1}{Z_{1} Z_{2} Z_{3}}\right)^{2 / 3}(d t+k)^{2}+\left(Z_{1} Z_{2} Z_{3}\right)^{1 / 3} h_{m n} d x^{m} d x^{n} \\
& +\left(\frac{Z_{2} Z_{3}}{Z_{1}^{2}}\right)^{1 / 3}\left(d x_{1}^{2}+d x_{2}^{2}\right)+\left(\frac{Z_{1} Z_{3}}{Z_{2}^{2}}\right)^{1 / 3}\left(d x_{3}^{2}+d x_{4}^{2}\right)+\left(\frac{Z_{1} Z_{2}}{Z_{3}^{2}}\right)^{1 / 3}\left(d x_{5}^{2}+d x_{6}^{2}\right),
\end{aligned}
$$

and the standard approach to their constructing starts by taking the four-dimensional spatial base metric $h_{m n}$ to be that of flat $\mathbb{R}^{4}$ [22, 9, 23, 6], 10, [7].

The corresponding "bubbling" solutions [2, 3] are obtained by replacing this flat $\mathbb{R}^{4}$ by a half-flat, four-dimensional "generalized hyper-Kähler" metric that is asymptotic to $\mathbb{R}^{4}$. By "generalized," we mean that one can use a hyper-Kähler base whose metric is 
allowed to change its overall sign in compact regions, thereby flipping the signature from +4 to -4 . The overall, eleven-dimensional metric is perfectly regular and Lorentzian essentially because any overall change of sign in the four-dimensional base metric is exactly compensated by sign changes in the warp factors $Z_{i}$. [16,2, 3 .

This opens up a huge array of new possibilities for constructing bubbling black holes and rings, because there are many, many hyper-Kähler metrics that are asymptotic to $\mathbb{R}^{4}$ but have compact regions where the metric changes its overall sign 6 . For practical reasons, the solutions of [1, 2, 3] were restricted to the simplest class of generalized hyper-Kähler geometries: Gibbons-Hawking (GH) metrics whose potential, $V$, is allowed to be negative. Mathematically, these metrics are extremely special: They constitute the complete family of four-dimensional hyper-Kähler metrics with a tri-holomorphic $U(1)$ isometry. The general BPS solutions with a positive-definite GH base were obtained in [4], and it is straightforward to adapt these solutions to generalized GH metrics that allow overall sign changes. In this paper we will work entirely within this class of solutions.

We therefore consider a four-dimensional base metric that has Gibbons-Hawking (GH) form:

$$
d s_{4}^{2}=V^{-1}(d \psi+\vec{A} \cdot d \vec{y})^{2}+V(d \vec{y} \cdot d \vec{y})
$$

where $\vec{y} \in \mathbb{R}^{3}$ and

$$
V=\sum_{j=1}^{N} \frac{q_{j}}{r_{j}}, \quad \vec{\nabla} \times \vec{A}=\vec{\nabla} V,
$$

with $r_{j} \equiv\left|\vec{y}-\vec{y}^{(j)}\right|$. In order for the GH metric to be regular, one must take $q_{j} \in \mathbb{Z}$ and for the metric to be asymptotic to that of flat $\mathbb{R}^{4}$ one must also impose

$$
q_{0} \equiv \sum_{j=1}^{N} q_{j}=1
$$

The GH metric has non-trivial two-cycles (the bubbles), $\Delta_{i j}$, defined by the fiber coordinate, $\psi$, and any line running between a pair of GH points, $\vec{y}^{(i)}$ and $\vec{y}^{(j)}$.

The background three-form potentials in the eleven-dimensional solution are defined via vector potentials in the four-dimensional GH base.

$$
\mathcal{A}=A^{(1)} \wedge d x_{1} \wedge d x_{2}+A^{(2)} \wedge d x_{3} \wedge d x_{4}+A^{(3)} \wedge d x_{5} \wedge d x_{6}
$$

6 Based on the physics of three-charge supertubes [8], one expects generalized hyper-Kähler metrics with $N$ regions of negative signature to be parameterized by $6 N$ functions of one variable [2]. 
Supersymmetry requires the "dipole field strengths:"

$$
\Theta^{(I)} \equiv d A^{(I)}+d\left(\frac{d t+k}{Z_{I}}\right)
$$

to be self-dual. One can easily show that if the base has a Gibbons-Hawking metric, then the $\Theta^{I}$ are given by

$$
\Theta^{(I)}=-\sum_{a=1}^{3}\left[\partial_{a}\left(V^{-1} K^{I}\right)\right]\left[(d \psi+A) \wedge d y^{a}+\frac{1}{2} V \epsilon_{a b c} d y^{b} \wedge d y^{c}\right] .
$$

where $K^{I}$ is harmonic in the $\mathbb{R}^{3}$ base of the GH space. To have completely non-singular solutions one must choose these to be sourced only at the singular points of $V$ :

$$
K^{I}=\sum_{j=1}^{N} \frac{k_{j}^{I}}{r_{j}}
$$

One then finds that the two-form fluxes through $\Delta_{i j}$ are given by:

$$
\Pi_{i j}^{(I)}=\left(\frac{k_{j}^{I}}{q_{j}}-\frac{k_{i}^{I}}{q_{i}}\right) .
$$

Having chosen the flux parameters, $k_{j}^{I}$, the solution is completely fixed by its asymptotics and by requiring the absence of any singularities and, in particular, the absence of singular sources. One introduces functions, $L^{I}$, and $M$ defined by:

$$
L_{I}=1-\frac{1}{2} C_{I J K} \sum_{j=1}^{N} \frac{k_{j}^{J} k_{j}^{K}}{q_{j}} \frac{1}{r_{j}}, \quad M=m_{0}+\frac{1}{12} C_{I J K} \sum_{j=1}^{N} \frac{k_{j}^{I} k_{j}^{J} k_{j}^{K}}{q_{j}^{2}} \frac{1}{r_{j}}
$$

with

$$
m_{0}=-\frac{1}{2} q_{0}^{-1} \sum_{j=1}^{N} \sum_{I} k_{j}^{I}=-\frac{1}{2} \sum_{j=1}^{N} \sum_{I} k_{j}^{I} .
$$

The warp factors, $Z_{I}$, are then given by:

$$
Z_{I}=L_{I}+\frac{1}{2} C_{I J K} V^{-1} K^{J} K^{K}
$$

and the angular momentum vector, $k$, in (2.1) is:

$$
k=\mu(d \psi+A)+\omega,
$$


with

$$
\mu=\frac{1}{6} V^{-2} C_{I J K} K^{I} K^{J} K^{K}+\frac{1}{2} V^{-1} K^{I} L_{I}+M,
$$

and $\omega$ defined by $\omega=\vec{\omega} \cdot d \vec{y}$, where

$$
\vec{\nabla} \times \vec{\omega}=V \vec{\nabla} M-M \vec{\nabla} V+\frac{1}{2}\left(K^{I} \vec{\nabla} L_{I}-L_{I} \vec{\nabla} K^{I}\right) .
$$

One needs to solve this equation without introducing Dirac-Misner strings into the metric and to do this it is convenient to introduce one forms, $\omega_{i j}$, associated to each pair of $\mathrm{GH}$ points. The simplest way to define these forms is to choose coordinates so that $\vec{y}=(x, y, z)$ and $\vec{y}^{(i)}=(0,0, a)$ and $\vec{y}^{(j)}=(0,0, b)$, with $a>b$, and then one sets:

$$
\omega_{i j} \equiv-\frac{\left(x^{2}+y^{2}+\left(z-a+r_{i}\right)\left(z-b-r_{j}\right)\right)}{(a-b) r_{i} r_{j}} d \phi,
$$

where $\tan \phi=y / x$. The importance of this form is that it has no Dirac strings. The desired non-singular solution to (2.15) may then be written as

$$
\vec{\omega}=\frac{1}{24} C_{I J K} \sum_{i, j=1}^{N} q_{i} q_{j} \Pi_{i j}^{(I)} \Pi_{i j}^{(J)} \Pi_{i j}^{(K)} \vec{\omega}_{i j},
$$

provided that the bubble equations are satisfied:

$$
\frac{1}{6} C_{I J K} \sum_{\substack{j=1 \\ j \neq i}}^{N} \Pi_{i j}^{(I)} \Pi_{i j}^{(J)} \Pi_{i j}^{(K)} \frac{q_{i} q_{j}}{r_{i j}}=-2\left(m_{0} q_{i}+\frac{1}{2} \sum_{I=1}^{3} k_{i}^{I}\right),
$$

for $i=1, \ldots, N$, and where $r_{i j} \equiv\left|\vec{y}^{(i)}-\vec{y}^{(j)}\right|$. The bubble equations are required to remove closed timelike curves (CTC's) in specific, "potentially dangerous" limitst. In general, the absence of CTC's requires that one ensure that the following are globally true:

$$
V Z_{I} \geq 0, \quad Z_{1} Z_{2} Z_{3} V-\mu^{2} V^{2} \geq 0
$$

However, in quite a number of examples one finds that the bubble equations suffice to guarantee the global absence of CTC's.

Finally, one should note that shifting $K^{I} \rightarrow K^{I}+c^{I} V$ for some constants, $c^{I}$, has a trivial action on the solution and so the parameters, $k_{j}^{I}$, have a gauge invariance:

$$
k_{j}^{I} \rightarrow k_{j}^{I}+q_{j} c^{I}
$$

All the physical quantities must be invariant under this transformation.

7 One can add a constant to $V$ in (2.3), and reduce the resulting smooth five-dimensional solution to a singular four-dimensional multi-black-hole solution of the type explored in [24,25]. The "bubble equations" are then equivalent to the "integrability conditions" of [24,25]. Other asymptotically four-dimensional configurations that are smooth in five-dimensions and are microstates of four-dimensional black holes have been explored in [26,27,28]. 


\subsection{The charges and angular momenta of the solution}

To isolate the charges of the solution one should first recall that if $V=\frac{1}{r}$, then the coordinate transformation that takes (2.2) to a standard polar form for $\mathbb{R}^{4}$ is $r=\frac{1}{4} \rho^{2}$, where $\rho$ is the standard radial coordinate. Imposing (2.4) means that we have $V \sim \frac{1}{r}$ at infinity, and so this gives us the proper radial coordinate asymptotically.

To obtain the electric charges measured at infinity, one simply needs to extract the coefficient of $\rho^{-2}$ in the expansion of the $Z_{I}$. It is elementary to see that:

$$
Q_{I}=-2 C_{I J K} \sum_{j=1}^{N} q_{j}^{-1} \tilde{k}_{j}^{J} \tilde{k}_{j}^{K}
$$

where

$$
\tilde{k}_{j}^{I} \equiv k_{j}^{I}-q_{j} N k_{0}^{I}, \quad \text { and } \quad k_{0}^{I} \equiv \frac{1}{N} \sum_{j=1}^{N} k_{j}^{I} .
$$

Note that $\tilde{k}_{j}^{I}$ is invariant under (2.20).

To read off the angular momenta one looks at the asymptotic behavior of $k$ in (2.13) and extracts the terms that fall-off as $\frac{1}{\rho^{2}} \sim \frac{1}{4 r}$. Indeed, it is easiest to get the result from the coefficient of $d \psi$, that is, from the function $\mu$. There are two types of such terms,

simple $\frac{1}{r}$ terms and the dipole terms arising from the expansion of $V^{-1} K^{I}$. Following [3], we introduce the dipoles

$$
\vec{D}_{j} \equiv \sum_{I} \tilde{k}_{j}^{I} \vec{y}^{(j)}, \quad \vec{D} \equiv \sum_{j=1}^{N} \vec{D}_{j}
$$

and then one can obtain the components of the angular momentum from:

$$
k \sim \frac{1}{4 \rho^{2}}\left(\left(J_{1}+J_{2}\right)+\left(J_{1}-J_{2}\right) \cos \theta\right) d \psi+\ldots
$$

where $\theta$ is the angle between $\vec{D}$ and $\vec{y}$. Expanding the function $\mu$, we find:

$$
\begin{gathered}
J_{R} \equiv J_{1}+J_{2}=\frac{4}{3} C_{I J K} \sum_{j=1}^{N} q_{j}^{-2} \tilde{k}_{j}^{I} \tilde{k}_{j}^{J} \tilde{k}_{j}^{K} \\
J_{L} \equiv J_{1}-J_{2}=8|\vec{D}| .
\end{gathered}
$$

While we have put modulus signs around $\vec{D}$ in $(2.26)$, one should note that it does have a meaningful orientation, and so we will sometimes consider $\vec{J}_{L}=8 \vec{D}$. 
These results appear to differ by some factors of two compared to those of [3]. This is because our conventions are those of [2], which use a different normalization of the two-form fields.

One can use the bubble equations to obtain another, rather more intuitive expression for $J_{1}-J_{2}$. One should first note that the right-hand side of the bubble equation, (2.18), may be written as $-\sum_{I} \tilde{k}_{i}^{I}$. Multiplying this by $\vec{y}^{(i)}$ and summing over $i$ yields:

$$
\begin{aligned}
\vec{J}_{L} \equiv 8 \vec{D} & =-\frac{4}{3} C_{I J K} \sum_{\substack{i, j=1 \\
j \neq i}}^{N} \Pi_{i j}^{(I)} \Pi_{i j}^{(J)} \Pi_{i j}^{(K)} \frac{q_{i} q_{j} \vec{y}^{(i)}}{r_{i j}} \\
& =-\frac{2}{3} C_{I J K} \sum_{\substack{i, j=1 \\
j \neq i}}^{N} q_{i} q_{j} \Pi_{i j}^{(I)} \Pi_{i j}^{(J)} \Pi_{i j}^{(K)} \frac{\left(\vec{y}^{(i)}-\vec{y}^{(j)}\right)}{\left|\vec{y}^{(i)}-\vec{y}^{(j)}\right|}
\end{aligned}
$$

where we have used the skew symmetry $\Pi_{i j}=-\Pi_{j i}$ to obtain the second identity. This result suggests that one should define an angular momentum flux vector associated with the $i j^{\text {th }}$ bubble:

$$
\vec{J}_{L i j} \equiv-\frac{4}{3} q_{i} q_{j} C_{I J K} \Pi_{i j}^{(I)} \Pi_{i j}^{(J)} \Pi_{i j}^{(K)} \hat{y}_{i j}
$$

where $\hat{y}_{i j}$ are unit vectors,

$$
\hat{y}_{i j} \equiv \frac{\left(\vec{y}^{(i)}-\vec{y}^{(j)}\right)}{\left|\vec{y}^{(i)}-\vec{y}^{(j)}\right|} .
$$

This means that the flux terms on the left-hand side of the bubble equation actually have a natural spatial direction, and once this is incorporated, it yields the contribution of the bubble to $J_{L}$.

\subsection{The simplest bubbled supertube}

For later convenience we summarize the properties of the solution with three GH points of charges $q_{1}=1, q_{2}=-Q$ and $q_{3}=+Q$ that corresponds to a zero-entropy black ring [2]. It is useful to define new, physical variables $d^{I}$ and $f^{I}$ (the $d^{I}$ are equal to the dipole charges of the ring):

$$
d^{I} \equiv 2\left(k_{2}^{I}+k_{3}^{I}\right), \quad f_{I} \equiv 2 k_{1}^{I}+\left(1+\frac{1}{Q}\right) k_{2}^{I}+\left(1-\frac{1}{Q}\right) k_{3}^{I}
$$

Note that $d^{I}$ and $f^{I}$ are invariant under (2.20).

The electric charges of the bubbled tube are:

$$
Q_{I}=C_{I J K} d^{J} f^{K}
$$


and the angular momenta are:

$$
\begin{aligned}
& J_{1}=-\frac{(Q-1)}{12 Q} C_{I J K} d^{I} d^{J} d^{K}+\frac{1}{2} C_{I J K} d^{I} d^{J} f^{K}, \\
& J_{2}=\frac{(Q-1)^{2}}{24 Q^{2}} C_{I J K} d^{I} d^{J} d^{K}+\frac{1}{2} C_{I J K} f^{I} f^{J} d^{K} .
\end{aligned}
$$

In particular, the angular momentum of the tube is:

$$
J_{T}=J_{2}-J_{1}=\frac{1}{2} C_{I J K}\left(f^{I} f^{J} d^{K}-d^{I} d^{J} f^{K}\right)+\left(\frac{3 Q^{2}-4 Q+1}{24 Q^{2}}\right) C_{I J K} d^{I} d^{J} d^{K}
$$

The radius, $R_{T}$, of the corresponding classical black ring, as measured in the $\mathbb{R}^{3}$ metric of the GH base, can be obtained from:

$$
J_{T}=4 R_{T}\left(d^{1}+d^{2}+d^{3}\right)
$$

\section{Bubbling black holes and rings with a large number of centers}

In [1] we constructed bubbled solutions corresponding to maximally-spinning (zeroentropy) BMPV black holes, or to maximally spinning BPS black rings. These solutions have a very large number of GH centers and a priori there to be rather little difference between bubbling a black hole and bubbling a black ring: the ring microstates have a blob of GH centers of zero total charge with a GH center away from the blob while the black hole microstates have all the centers in one blob of net GH charge one. We will see that this apparently small difference can very significantly influence the solution of the bubble equations The purpose of this section is to explain in more detail the construction of microstates in [1], and to investigate numerically the structure of the distribution of GH centers inside the blobs.

\subsection{Microstates of maximally spinning BMPV black holes}

We first consider a configuration of $N$ GH centers that lie is a single "blob" and take all these centers to have roughly the same flux parameters, to leading order in $N$. To argue that such a blob corresponds to a BMPV black hole, we first need to show that $J_{1}=J_{2}$. If the overall configuration has three independent $\mathbb{Z}_{2}$ reflection symmetries then this is trivial because the $\vec{D}_{j}$ will then come in equal and opposite pairs, and so one has $J_{L}=0$. More 
generally, for a "random" distribution 8 the vectors $\hat{y}_{i j}$ will point in "random" directions and so the $\vec{J}_{L i j}$ will generically cancel one another at leading order in $N$. The only way to generate a non-zero value of $J_{L}$ is to bias the distribution so that there are more positive centers in one region and more negative ones in another. This is essentially what happens in the microstate solutions constructed and analyzed by [13, 14, 15, 16]. However, a single generic blob will have $J_{1}-J_{2}$ small compared to $\left|J_{1}\right|$ and $\left|J_{2}\right|$.

To compute the other properties of such a blob, we will simplify things by taking $N=2 M+1$ to be odd, and assume that $q_{j}=(-1)^{j+1}$. Using the gauge invariance, we can take all of $k_{i}^{I}$ to be positive numbers, and we will assume that they have small variations about their mean value:

$$
k_{j}^{I}=k_{0}^{I}(1+\mathcal{O}(1)),
$$

where $k_{0}^{I}$ is defined in (2.22). The charges are given by:

$$
\begin{aligned}
Q_{I} & =-2 C_{I J K} \sum_{j} q_{j}^{-1}\left(k_{j}^{J}-q_{j} N k_{0}^{J}\right)\left(k_{j}^{K}-q_{j} N k_{0}^{K}\right) \\
& =-2 C_{I J K}\left(\sum_{j} q_{j}^{-1} k_{j}^{J} k_{j}^{K}-N k_{0}^{J} \sum_{j} k_{j}^{K}-N k_{0}^{K} \sum_{j} k_{j}^{J}+N^{2} k_{0}^{J} k_{0}^{K} \sum_{j} q_{j}\right) \\
& =2 C_{I J K}\left(N^{2} k^{J} k^{K}-\sum_{j} k_{j}^{J} k_{j}^{K} q_{j}^{-1}\right) \\
& \approx 2 C_{I J K}\left(N^{2}+\mathcal{O}(1)\right) k_{0}^{J} k_{0}^{K}
\end{aligned}
$$

where we used (3.1) and the fact that $\left|q_{i}\right|=1$ only in the last step. In the large $N$ limit, for M theory on $T^{6}$ we have

$$
Q_{1} \approx 4 N^{2} k^{2} k^{3}+\mathcal{O}(1), \quad Q_{2} \approx 4 N^{2} k^{1} k^{3}+\mathcal{O}(1), \quad Q_{3} \approx 4 N^{2} k^{1} k^{3}+\mathcal{O}(1)
$$

We can make a similar computation for the angular momenta:

$$
\begin{gathered}
J_{R}=\frac{4}{3} C_{I J K} \sum_{j} q_{j}^{-2}\left(k_{j}^{I}-q_{j} N k_{0}^{I}\right)\left(k_{j}^{J}-q_{j} N k_{0}^{J}\right)\left(k_{j}^{K}-q_{j} N k_{0}^{K}\right) \\
=\frac{4}{3} C_{I J K}\left(\sum_{j} q_{j}^{-2} k_{j}^{I} k_{j}^{J} k_{j}^{K}-3 N k_{0}^{I} \sum_{j} q_{j}^{-1} k_{j}^{J} k_{j}^{K}\right. \\
\left.\quad+3 N^{2} k_{0}^{I} k_{0}^{J} \sum_{j} k_{j}^{K}-N^{3} k_{0}^{I} k_{0}^{J} k_{0}^{K} \sum_{j} q_{j}\right) \\
\approx \frac{4}{3} C_{I J K}\left(N-3 N+3 N^{3}-N^{3}+\mathcal{O}(N)\right) k_{0}^{I} k_{0}^{J} k_{0}^{K},
\end{gathered}
$$

8 Such a distribution must, of course, satisfy the bubble equations, (2.18), but this will still allow a sufficiently random distribution of GH points. 
where we used the fact that, for a "well behaved" distribution of positive $k_{i}^{I}$ with $\left|q_{j}\right|=1$, one has:

$$
\sum_{i} q_{i}^{-1} k_{i}^{J} k_{i}^{K}=\sum_{i} q_{i} k_{i}^{J} k_{i}^{K} \approx k_{0}^{J} k_{0}^{K}, \quad \sum_{i} k_{i}^{I} k_{i}^{J} k_{i}^{K} \approx N k_{0}^{I} k_{0}^{J} k_{0}^{K}
$$

For M theory on $T^{6}$ we simply have:

$$
J_{R} \approx 16 N^{3} k^{1} k^{2} k^{3}+O(N)
$$

For large $N$ we therefore have, at leading order:

$$
J_{1}^{2} \approx J_{2}^{2} \approx \frac{1}{4} J_{R}^{2} \approx Q_{1} Q_{2} Q_{3}
$$

and so, in the large- $N$ limit, these microstates always correspond to a maximally spinning BMPV black hole. Indeed, we have

$$
\frac{J_{R}^{2}}{4 Q_{1} Q_{2} Q_{3}}-1 \sim O\left(\frac{1}{N^{2}}\right) .
$$

Interestingly enough, the value of $J_{R}$ is slightly bigger than $\sqrt{4 Q_{1} Q_{2} Q_{3}}$. However, this is not a problem because in the classical limit this correction vanishes. Moreover, it is possible to argue that a classical black hole with zero horizon area will receive higherorder curvature corrections, that usually increase the horizon area; hence a zero-entropy configuration will have $J_{R}$ slightly larger then the maximal classically allowed value, by an amount that vanishes in the large $N$ (classical) limit.

\subsection{Zero-entropy black ring (supertube) microstates}

The next simplest configuration to consider is one in which one starts with the blob considered above and then moves a single GH point of charge +1 out to a very large distance from the blob. That is, one considers a blob of total GH charge zero with a single very distant point of GH-charge +1 . Since one now has a strongly "biased" distribution of GH charges one should now expect $J_{1}-J_{2} \neq 0$.

Again we will assume $N$ to be odd, and take the a GH charge distribution to be

$q_{j}=(-1)^{j+1}$, with the distant charge being the $N^{\text {th }} \mathrm{GH}$ charge. We will also fix the gauge by taking $k_{N}^{I} \equiv 0$. The blob therefore has $\frac{1}{2}(N-1)$ points of GH charge \pm 1 and at large scales one might expect it to resemble the three-point solution described above with $Q=\frac{1}{2}(N-1)$. We will show that this is exactly what happens in the large- $N$ limit. 
To have the $N^{\text {th }}$ GH charge far from the blob means that all the two-cycles, $\Delta_{j N}$ must support a very large flux compared to the fluxes on the $\Delta_{i j}$ for $i, j<N$. To achieve this we therefore take:

$$
k_{j}^{I}=a_{0}^{I}(1+\mathcal{O}(1)), \quad j=1, \ldots, N-1, \quad k_{N}^{I}=-b_{0}^{I} N .
$$

where $b_{0}^{I}$ is independent of $j$ and

$$
a_{j}^{I} \equiv k_{j}^{I}=a_{0}^{I}(1+\mathcal{O}(1)), \quad j=1, \ldots, N-1,
$$

with

$$
a_{0}^{I} \equiv \frac{1}{(N-1)} \sum_{j=1}^{N-1} a_{j}^{I} .
$$

We also assume that $a_{0}^{I}$ and $b_{0}^{I}$ are of the same order. The fluxes of this configuration are then:

$$
\Pi_{i j}^{(I)}=\left(\frac{a_{j}^{I}}{q_{j}}-\frac{a_{i}^{I}}{q_{i}}\right), \quad \Pi_{i N}^{(I)}=-\Pi_{N i}^{(I)}=-\left(\frac{a_{i}^{I}}{q_{i}}+N b_{0}^{I}\right), \quad i, j=1, \ldots, N-1 .
$$

For this configuration one has:

$$
\begin{aligned}
& k_{0}^{I}=\frac{(N-1)}{N} a_{0}^{I}-b_{0}^{I}, \quad \tilde{k}_{N}^{I}=-(N-1) a_{0}^{I}, \\
& \tilde{k}_{j}^{I}=a_{j}^{I}+q_{j}\left(N b_{0}^{I}-(N-1) a_{0}^{I}\right), \quad j=1, \ldots, N-1 .
\end{aligned}
$$

Motivated by the bubbling black ring of [2], define the physical parameters:

$$
d^{I} \equiv 2(N-1) a_{0}^{I}, \quad f^{I} \equiv(N-1) a_{0}^{I}-2 N b_{0}^{I} .
$$

Keeping only the terms of leading order in $N$ in (2.21) and (2.25), one finds:

$$
Q_{I}=C_{I J K} d^{J} f^{K}, \quad J_{1}+J_{2}=\frac{1}{2} C_{I J K}\left(d^{I} d^{J} f^{K}+f^{I} f^{J} d^{K}\right)-\frac{1}{24} C_{I J K} d^{I} d^{J} d^{K} .
$$

Since the $N^{\text {th }}$ point is far from the blob, we can take $r_{i N} \approx r_{0}$ and then the $N^{\text {th }}$ bubble equation reduces to:

$$
\frac{1}{6} C_{I J K} \sum_{j=1}^{N-1}\left(\frac{a_{j}^{I}}{q_{j}}+N b_{0}^{I}\right)\left(\frac{a_{j}^{J}}{q_{j}}+N b_{0}^{J}\right)\left(\frac{a_{j}^{K}}{q_{j}}+N b_{0}^{K}\right) \frac{q_{j}}{r_{0}}=(N-1) \sum_{I} a^{I} .
$$


To leading order in $N$ this means that the distance from the blob to the $N^{\text {th }}$ point, $r_{0}$, in the GH space is given by:

$$
r_{0} \approx \frac{1}{2} N^{2}\left[\sum_{I} a^{I}\right]^{-1} C_{I J K} a_{0}^{I} b_{0}^{J} b_{0}^{K}=\frac{1}{32}\left[\sum_{I} d^{I}\right]^{-1} C_{I J K} d^{I}\left(2 f^{J}-d^{J}\right)\left(2 f^{K}-d^{K}\right) .
$$

Finally, considering the dipoles, (2.23), it is evident that to leading order in $N, \vec{D}$ is dominated by the contribution coming from the $N^{\text {th }}$ point and that:

$$
\begin{aligned}
J_{1}-J_{2} & =8|\vec{D}|=8 N\left(\sum_{I} a_{0}^{I}\right) r_{0}=4 N^{3} C_{I J K} a_{0}^{I} b_{0}^{J} b_{0}^{K} \\
& =\frac{1}{8} C_{I J K} d^{I}\left(2 f^{J}-d^{J}\right)\left(2 f^{K}-d^{K}\right) .
\end{aligned}
$$

One can easily verify that these results perfectly match the properties of the threecenter bubbled supertube constructed in [2], and summarized above. Thus the blob considered here has exactly the same size, angular momenta, charges and dipole charges as a zero-entropy black ring.

\subsection{Numerical investigation of bubbling solutions}

It is extremely instructive to solve the bubble equations numerically for fairly large values of $N$ because one discovers some interesting qualitative results. Most particularly, the interior structures of bubbled black holes and bubbled black rings is very different, at least when all the flux parameters are equal. The black ring blob has zero total GH charge, and the individual GH charges form tight, neutral clusters that are fairly broadly spaced. The black hole blob on the other hand has a net GH charge, which prevents the formation of neutral clusters everywhere. In fact the GH charges do form broadly-spaced tight, neutral clusters but only in the outer parts of the blob. The excess GH charge can be found in the deep interior of the blob, where it is "screened" by the neutral clusters.

We solved numerically the bubble equations for a very large number of GH centers lying on the same axis, with alternating charges $q_{j}= \pm 1$. To get the "ring blob" we took the flux parameters $k_{i}$ to be equal on the first $(N-1)$ points and adjusted the flux parameter on the last point so as to move it away from the blob. We generically found the solution to consist of tightly bound dipoles in the blob and that the spacing between two neighboring dipoles was at least an order of magnitude larger that the size of an individual dipole. The ratio of the separation of neighboring dipoles and the size of a dipole grows larger as the $N^{\text {th }}$ "far away" GH point is moved further and further from the blob. Figure 2 shows a typical set of spacings between successive GH points. There are two branches to this graph: The size of dipoles and the space between neighboring dipoles. 


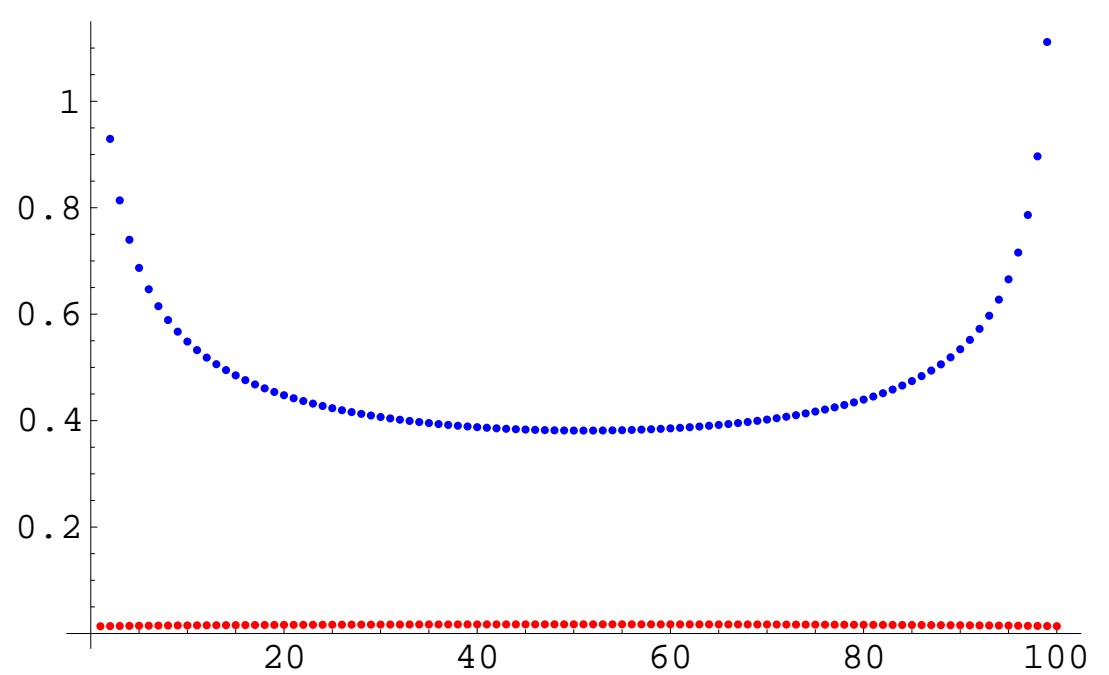

Fig. 2: A ring distribution for $N=201$ points in which the $j^{\text {th }}$ point has $G H$ charge $(-1)^{j+1}$. There is a fixed flux parameter, $k=1$, on first $(N-1)$ points, and a flux parameter $20 k$ on the $N^{\text {th }}$ point. The width of the ring distribution (the distance from first to $(N-1)^{\text {th }}$ point $)$ is 49.09. The distance from $(N-1)^{\text {th }}$ to $N^{\text {th }}$ point is 376.41. This graph shows distances between successive GH points. The lower curve shows the spacing between the $(2 j-1)^{\text {th }}$ point and the $(2 j)^{\text {th }}$ point, $j=1, \ldots, 100$, forming $(+-)$ dipole pairs. The upper curve shows the spacing between the $(2 j)^{\text {th }}$ point and the $(2 j+1)^{\text {th }}$ point, $j=1, \ldots, 99$, representing the separations of neighboring dipoles. The "far away" point is not shown. Note that the entire distribution shows a set of 100 close (+ -) dipoles. All distances are measured using the flat $\mathbb{R}^{3}$ part of the GH metric.

To get a BMPV blob we took all the flux parameters to be equal and sought solutions where the GH charges alternated along a symmetry axis in $\mathbb{R}^{3}$. All the solutions we found had a further reflection symmetry in the distribution of GH charges, and this symmetry guarantees that $\vec{D} \equiv 0$, and so $J_{1}=J_{2}$. The distribution of spaces between GH points is completely different from that of the "ring blob." By construction, the outermost points had GH charge +1 and we found that the charges formed close dipoles with their nearest neighbors. Thus the outer part of the distribution tries to form $(+-)$ dipoles on the left and $(-+)$ dipoles on the right. This cannot be sustained in the interior and so the dipole length grows and the charges become evenly-spaced towards the middle of the configuration. The excess GH charge of the blob is thus in the middle and it tends to separate the nearby dipoles. Figure 3 shows a typical set of spacings between successive GH points in a BMPV blob. Again there are two branches, one for the $(+-)$-pairs and another for the $(-+)$-pairs. These two branches cross in the center. 


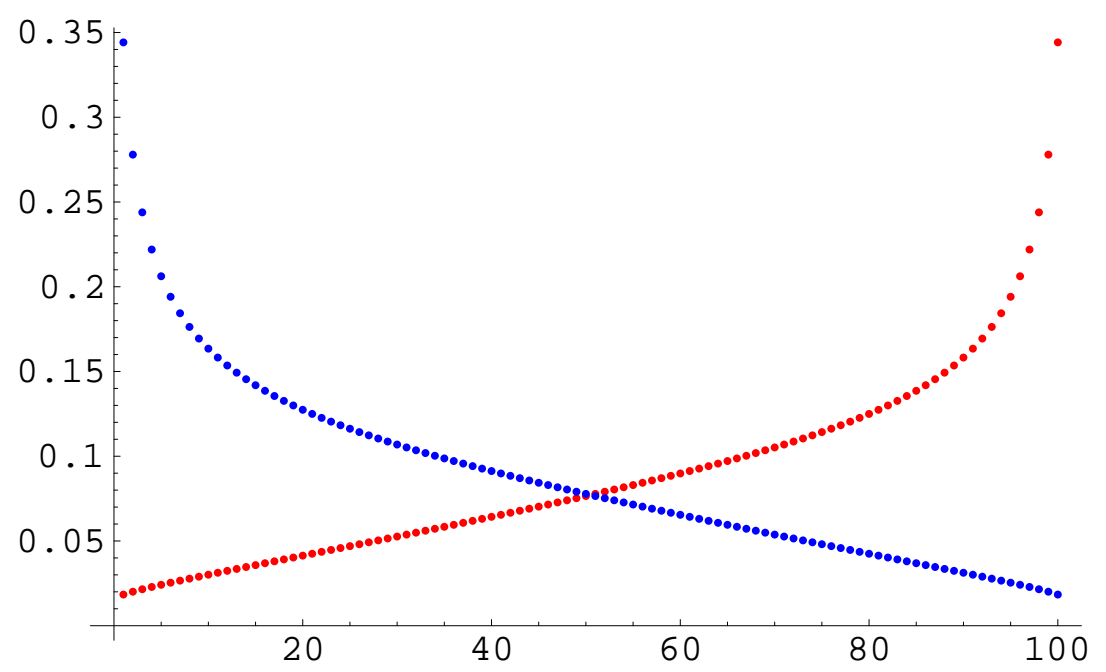

Fig. 3: A BMPV blob with $N=201$ points and all flux parameters equal. The $j^{\text {th }}$ point has $G H$ charge $(-1)^{j+1}$. This graph shows distances between neighboring GH points. The curve from bottom-left to top-right shows the spacing between the $(2 j-1)^{\text {th }}$ point and the $(2 j)^{\text {th }}$ point, a $(+-)$-pair, $j=1, \ldots, 100$. The curve from top-left to bottom-right shows the spacing between the $(2 j)^{\text {th }}$ point and the $(2 j+1)^{\text {th }}$ point, a (-+)-pair, $j=1, \ldots, 100$. Note how the points are evenly spaced in the middle and form a more diffuse "gas" of close dipoles of opposite orientation at the edges.

One important consequence of this is that a black-ring blob and a black-hole blob are very different from each other, despite having similar flux parameters. Moving a single GH point out of a large blob, might naively appear to be a small perturbation; it can however result in a huge "phase transition" in the interior structure of the blob. In particular, moving a single +1 point from the edge of the "BMPV blob" in Figure 3 should result in a transition to the distribution shown in Figure 2. We will see an example of this in Section 6.

\section{Mergers and microstates}

Having found some of the microstates corresponding to the maximally-spinning BMPV black hole and to a supertube (zero-entropy black ring), we now revisit the merger process of a black ring and a black hole from the perspective of microstates. We begin by recalling some of the key results of [19]. 


\subsection{Classical mergers and their bubbled counterparts}

Consider the $U(1)$-invariant configurations of a black ring and a black hole in which the black hole lies on the axis of the ring, but offset "vertically" from the center. These solutions were first obtained and studied in [19]. In particular, for fixed macroscopic charges on the ring and black hole, it was shown how the embedding radius of the ring must vary as one varies the offset. In this process, one component, $J_{1}$, of the angular momentum in the ring plane is conserved, while the other component, $J_{2}$, changes. The merger of the black ring with the black hole occurs precisely when $J_{L} \equiv J_{1}-J_{2}=0$.

Generically such a merger is thermodynamically irreversible and results in a BMPV black hole $\left(J_{1}=J_{2}\right)$ with a total horizon area that exceeds the sum of the areas of the two original horizons. There is, however, one possible way to make a reversible merger: The initial black hole and black ring have to have vanishing horizon area and these could merge to yield a larger, maximally-spinning (zero-horizon-area) black hole provided that two conditions are met. First, the ring must merge with the black hole at the equator of the black hole, so that at the point of merger the solution has a $U(1) \times U(1)$ invariance. Secondly, the charge vectors of the ring and of the black hole must be parallel. Any other merger will result in an entropy increase.

One can also study the merger by considering a $U(1) \times U(1)$ invariant solution describing a black ring with a black hole in the center [23,6, 7]. As the ring is made smaller and smaller by, for example, decreasing its angular momentum, it eventually merges into the black hole. At the point of merger, this solution is identical to the merger described above with the black ring grazing the black-hole horizon. Hence this $U(1) \times U(1)$ invariant solution can be used to study mergers where the black ring grazes the black hole horizon at the point of merger; all the reversible mergers and some of the irreversible mergers belong to this class.

In the previous section we have seen how to create bubbled solutions corresponding to zero-entropy black rings and maximally-spinning black holes. The generic bubbled solutions with GH base have a $U(1)$ symmetry corresponding to $J_{R} \equiv J_{1}+J_{2}$ and if the GH points all lie on an axis then the solution is $U(1) \times U(1)$ invariant. Conversely [18], all $U(1) \times U(1)$ invariant, four-dimensional hyper-Kähler metrics must be of $\mathrm{GH}$ form. We can therefore study the merger of bubbled microstates by constructing $U(1) \times U(1)$ invariant bubbling solutions describing a black ring with a black hole in the center. By decreasing some of the flux parameters of the solution one can decrease the radius of the 
bubbling black ring and merge it into the bubbling black hole to create a larger bubbling black hole.

In this section we consider a bubbling black hole with a very large number of GH centers, sitting at the center of the simplest bubbled supertube [2], generated by a pair of GH points $\mathrm{l}$. We expect two different classes of merger solution depending upon whether the flux parameters on the bubbled black hole and bubbled black ring are parallel or not. These correspond to reversible and irreversible mergers respectively. We will examine both situations in some detail, particularly when all the GH points lie on an axis and in which the solution has a $U(1) \times U(1)$ symmetry, and indeed find that reversible and irreversible mergers of microstates have very different physics. First, however, we will establish some general results about the charges and angular momenta of the bubbled solutions that describe a bubbled black ring of two GH centers with a BMPV black-hole blob at the center.

\subsection{Some exact results}

We begin by seeing what may be deduced with no approximations whatsoever. Our purpose here is to separate all the algebraic formulae for charges and angular momenta into those associated with the black hole foam and those associated with the bubbled supertube. We will consider a system of $N$ GH points in which the first $N-2$ points will be considered to be a blob and the last two points will have $q_{N-1}=-Q$ and $q_{N}=Q$. The latter two points can then be used to define a bubbled black ring.

Let $\hat{k}_{0}^{I}$ denote the average of the flux parameters over the first $(N-2)$ points:

$$
\hat{k}_{0}^{I} \equiv \frac{1}{(N-2)} \sum_{j=1}^{N-2} k_{j}^{I},
$$

and introduce $k$-charges that have a vanishing average over the first $(N-2)$ points:

$$
\hat{k}_{j}^{I} \equiv k_{j}^{I}-(N-2) q_{j} \hat{k}_{0}^{I}, \quad j=1, \ldots, N-2 .
$$

9 Of course it is straightforward to generalize our analysis to the situation where both the supertube and the black hole have a large number of GH centers. However, the analysis is simpler and the numerical stability is better for mergers in which the supertube is composed of only two points, and we have therefore focused on this. 
We also parameterize the last two $k^{I}$-charges in exactly the same manner as for the bubbled supertube (see (2.30)):

$$
d^{I} \equiv 2\left(k_{N-1}^{I}+k_{N}^{I}\right), \quad f^{I} \equiv 2(N-2) \hat{k}_{0}^{I}+\left(1+\frac{1}{Q}\right) k_{N-1}^{I}+\left(1-\frac{1}{Q}\right) k_{N}^{I} .
$$

One can easily show that the charge 2.21) decomposes into

$$
Q_{I}=\widehat{Q}_{I}+C_{I J K} d^{J} f^{K}
$$

where

$$
\widehat{Q}_{I} \equiv-2 C_{I J K} \sum_{j=1}^{N-2} q_{j}^{-1} \hat{k}_{j}^{J} \hat{k}_{j}^{K}
$$

The $\widehat{Q}_{I}$ are simply the charges of the black-hole blob, made of the first $(N-2)$ points. The second term in (4.4) is exactly the expression, (2.31), for the charges of a bubbled supertube with $\mathrm{GH}$ centers of charges $+1,-Q$ and $Q$ and $k$-charges $(N-2) \hat{k}_{0}^{I}, k_{N-1}^{I}$ and $k_{N}^{I}$, respectively. Thus the charge of this configuration decomposes exactly as if it were a black-hole blob of $(N-2)$ centers and a bubbled supertube.

There is a similar result for the angular momentum, $J_{R}$. One can easily show that:

$$
J_{R}=\widehat{J}_{R}+d^{I} \widehat{Q}_{I}+j_{R}
$$

where

$$
\hat{J}_{R} \equiv \frac{4}{3} C_{I J K} \sum_{j=1}^{N-2} q_{j}^{-2} \hat{k}_{j}^{I} \hat{k}_{j}^{J} \hat{k}_{j}^{K}
$$

and

$$
j_{R} \equiv \frac{1}{2} C_{I J K}\left(f^{I} f^{J} d^{K}+f^{I} d^{J} d^{K}\right)-\frac{1}{24}\left(1-Q^{-2}\right) C_{I J K} d^{I} d^{J} d^{K} .
$$

The term, $\hat{J}_{R}$, is simply the right-handed angular momentum of the black-hole blob made from $N-2$ points. The "ring" contribution to the angular momentum, $j_{R}$, agrees precisely with $J_{1}+J_{2}$ given by (2.32) and (2.33) for an isolated bubbled supertube. The cross term, $d^{I} \widehat{Q}_{I}$ is represents the interaction of the flux of the bubbled ring and the charge of the black-hole blob. This interaction term is exactly the same as that found in [6,7, 19] for a concentric black hole and black ring.

Thus, as far as the charges and $J_{R}$ are concerned, the complete system is behaving as though it were a black-hole blob of $(N-2)$ points interacting with a bubbled supertube defined by the points with GH charges $\pm Q$ and a single point with $\mathrm{GH}$ charge +1 
replacing the black-hole blob. Note that no approximations were made in the foregoing computations, and the results are true independent of the locations of the GH charges.

To make further progress we need to make some assumptions about the configuration of the points. Suppose, for the moment, that all the GH charges lie on the $z$-axis at points $z_{i}$ with $z_{i}<z_{i+1}$. In particular, the $\mathrm{GH}$ charges, $-Q$ and $+Q$, are located at $z_{N-1}$ and $z_{N}$ respectively.

With this ordering of the GH points, the expression for $\vec{J}_{L}$ collapses to:

$$
J_{L}=\frac{4}{3} C_{I J K} \sum_{1 \leq i<j \leq N} q_{i} q_{j} \Pi_{i j}^{(I)} \Pi_{i j}^{(J)} \Pi_{i j}^{(K)}
$$

This expression can then be separated, just as we did for $J_{R}$, into a black-hole blob component, a ring component, and interaction cross-terms. To that end, define the lefthanded angular momentum of the blob to be:

$$
\widehat{J}_{L}=\frac{4}{3} C_{I J K} \sum_{1 \leq i<j \leq N-2} q_{i} q_{j} \Pi_{i j}^{(I)} \Pi_{i j}^{(J)} \Pi_{i j}^{(K)}
$$

Note that

$$
\Pi_{i j}^{(I)} \equiv\left(\frac{k_{j}^{I}}{q_{j}}-\frac{k_{i}^{I}}{q_{i}}\right)=\left(\frac{\hat{k}_{j}^{I}}{q_{j}}-\frac{\hat{k}_{i}^{I}}{q_{i}}\right), \quad 1 \leq i, j \leq N-2
$$

and so this only depends upon the fluxes in the blob.

The remaining terms in (4.9) may then be written in terms of $\hat{k}_{j}^{I}$, $d^{I}$ and $f^{I}$ defined in (4.2) and (4.3). In particular, there are terms that depend only upon $d^{I}$ and $f^{I}$, and then there are terms that are linear, quadratic and cubic in $\hat{k}_{j}^{I}$ (and depend upon $d^{I}$ and $\left.f^{I}\right)$. The linear terms vanish because $\sum_{j=1}^{N-2} \hat{k}_{j}^{I} \equiv 0$, the quadratic terms assemble into $\widehat{Q}_{I}$ of (4.5) and the cubic terms assemble into $\hat{J}_{R}$ of (4.7). The terms proportional to (4.7) cancel between the terms with $j=N-1$ and $j=N$, and one is left with:

$$
J_{L}=\widehat{J}_{L}-d^{I} \widehat{Q}_{I}+j_{L}
$$

where $j_{L}$ is precisely the angular momentum, $J_{T}$, of the tube:

$$
j_{L} \equiv \frac{1}{2} C_{I J K}\left(d^{I} f^{J} f^{K}-f^{I} d^{J} d^{K}\right)+\left(\frac{3 Q^{2}-4 Q+1}{24 Q^{2}}\right) C_{I J K} d^{I} d^{J} d^{K}
$$


Observe that (4.8) and (4.12) are exactly the angular momenta of the simplest bubbled supertube, (2.32) and (2.33). Again we see the cross-term from the interaction of the ring dipoles and the electric charge of the blob. Indeed, combining (4.6) and (4.11), we obtain:

$$
J_{1}=\widehat{J}_{1}+j_{1}, \quad J_{2}=\widehat{J}_{2}+j_{2}+d^{I} \widehat{Q}_{I},
$$

which is exactly how the angular momenta of the classical ring-hole solution decompose [19]. In particular, the term coming from the interaction of the ring dipole moment to the black hole charge only contributes to $J_{2}$.

The results obtained above are independent of whether the blob of $N-2$ points is a BMPV black-hole blob, or a more generic configuration. However, to study mergers, we will explore from now on configurations in which the blob is a black-hole microstate, with $\widehat{J}_{L}=0$. The end result of the merger process is also a BMPV black hole microstate, and so $J_{L}=0$. Therefore, the exact merger condition is simply:

$$
\begin{aligned}
\Omega & \equiv \frac{1}{2} C_{I J K}\left(d^{I} f^{J} f^{K}-f^{I} d^{J} d^{K}\right)+\left(\frac{3 Q^{2}-4 Q+1}{24 Q^{2}}\right) C_{I J K} d^{I} d^{J} d^{K}-d^{I} \widehat{Q}_{I} \\
& =0 .
\end{aligned}
$$

Using (2.34), this may be written:

$$
J_{T}-d^{I} \widehat{Q}_{I}=0,
$$

which is precisely the condition obtained in [19] for a classical black ring to merge with a black hole at its equator.

One should note that the argument that led to the expressions (4.11) and (4.12), and the exact merger condition, (4.14), apply far more generally. In particular we only needed the fact that the unit vectors, $\hat{y}_{i j}$, defined (2.29), are all parallel for $j=N-1$ and $j=N$. This is approximately true in may contexts, and most particularly if the line between the $(N-1)^{\text {th }}$ and $N^{\text {th }}$ points runs through the blob and the width of the blob is small compared to the distance to these two points.

One should also not be surprised by the generality of the result in equation (4.12). The angular momentum, $J_{T}$, is an intrinsic property of a black ring, and hence for a zeroentropy black ring, $J_{T}$ can only depend on the $d$ 's and f's, and cannot depend on the black hole charges (that is, the $\hat{k}_{j}^{I}$ ). Therefore, we could have obtained (4.12) by simply setting the black hole charge to zero, and then reading off $J_{T}$ from the simplest bubbling black ring solution [2] discussed in sub-section 2.3. Hence, one should think about the expression of $J_{T}$ in (2.34) as a universal relation between intrinsic properties of the bubbled ring: $J_{T}, d^{I}$ and $f^{I}$. 


\subsection{Some simple approximations}

We now return to a general distribution of GH points, but we will assume that the two "exceptional points" (the $(N-1)^{\text {th }}$ and $N^{\text {th }}$ points) are close together but very far from the black-hole blob of the remaining $(N-2)$ points. Set up coordinates in the geometric center of the black-hole blob, i.e. choose the origin so that

$$
\sum_{i=1}^{N-2} \vec{r}_{i}=0 .
$$

Let $r_{0} \equiv\left|\vec{r}_{N-1}\right|$ be the distance from the geometric center of the blob to the first exceptional point, and let $\hat{r}_{0}$ be the unit vector in that direction. The vector, $\vec{\Delta} \equiv \vec{r}_{N}-\vec{r}_{N-1}$, defines the width of the ring. We will assume that the magnitudes $\Delta \equiv|\vec{\Delta}|$ and $r_{j} \equiv\left|\vec{r}_{j}\right|$ are small compared to $r_{0}$. We will also need the first terms of the multipole expansions:

$$
\begin{aligned}
\frac{1}{\left|\vec{r}_{N-1}-\vec{r}_{j}\right|} & =\frac{1}{r_{0}}+\frac{\vec{r}_{j} \cdot \hat{r}_{0}}{r_{0}^{2}}+\ldots \\
\frac{1}{\left|\vec{r}_{N}-\vec{r}_{j}\right|} & =\frac{1}{r_{0}}+\frac{\left(\vec{r}_{j}-\vec{\Delta}\right) \cdot \hat{r}_{0}}{r_{0}^{2}}+\ldots
\end{aligned}
$$

For simplicity, we will also assume that the two "exceptional points" are co-linear with the origin so that

$$
r_{N} \equiv\left|\vec{r}_{N}\right|=r_{0}+\Delta .
$$

The last two bubble equations are then:

$$
\begin{gathered}
\frac{\gamma}{\Delta}-\sum_{j=1}^{N-2} \frac{q_{j} \alpha_{j}}{\left|\vec{r}_{N}-\vec{r}_{j}\right|}=\sum_{I}\left(N Q k_{0}^{I}-k_{N}^{I}\right), \\
-\frac{\gamma}{\Delta}+\sum_{j=1}^{N-2} \frac{q_{j} \beta_{j}}{\left|\vec{r}_{N-1}-\vec{r}_{j}\right|}=-\sum_{I}\left(N Q k_{0}^{I}+k_{N-1}^{I}\right)
\end{gathered}
$$

where $k_{0}^{I}$ is given in (2.22) and

$$
\begin{gathered}
\alpha_{j} \equiv \frac{1}{6} Q C_{I J K} \Pi_{j N}^{(I)} \Pi_{j N}^{(J)} \Pi_{j N}^{(K)} \\
=\frac{1}{6} Q C_{I J K}\left(\frac{k_{N}^{I}}{Q}-\frac{k_{j}^{I}}{q_{j}}\right)\left(\frac{k_{N}^{J}}{Q}-\frac{k_{j}^{J}}{q_{j}}\right)\left(\frac{k_{N}^{K}}{Q}-\frac{k_{j}^{K}}{q_{j}}\right), \\
\beta_{j} \equiv \frac{1}{6} Q C_{I J K} \Pi_{j(N-1)}^{(I)} \Pi_{j(N-1)}^{(J)} \Pi_{j(N-1)}^{(K)} \\
=-\frac{1}{6} Q C_{I J K}\left(\frac{k_{N-1}^{I}}{Q}+\frac{k_{j}^{I}}{q_{j}}\right)\left(\frac{k_{N-1}^{J}}{Q}+\frac{k_{j}^{J}}{q_{j}}\right)\left(\frac{k_{N-1}^{K}}{Q}+\frac{k_{j}^{K}}{q_{j}}\right),
\end{gathered}
$$




$$
\gamma \equiv \frac{1}{6} Q^{2} C_{I J K} \Pi_{(N-1) N}^{(I)} \Pi_{(N-1) N}^{(J)} \Pi_{(N-1) N}^{(K)}=\frac{1}{48} Q^{-1} C_{I J K} d^{I} d^{J} d^{K} .
$$

It is also convenient to introduce

$$
\alpha_{0} \equiv \sum_{j=1}^{N-2} q_{j} \alpha_{j}, \quad \beta_{0} \equiv \sum_{j=1}^{N-2} q_{j} \beta_{j}
$$

If one adds (4.19) and (4.20) then the terms involving $\gamma$ cancel and using (4.17) one then obtains:

$$
\sum_{j=1}^{N-2} q_{j}\left[\alpha_{j}\left(\frac{1}{r_{0}}+\frac{\left(\vec{r}_{j}-\vec{\Delta}\right) \cdot \hat{r}_{0}}{r_{0}^{2}}\right)-\beta_{j}\left(\frac{1}{r_{0}}+\frac{\vec{r}_{j} \cdot \hat{r}_{0}}{r_{0}^{2}}\right)\right]=\frac{1}{2} \sum_{I} d^{I} .
$$

One now needs to perform the expansions with some care. Introduce the flux vector:

$$
X^{I} \equiv 2 f^{I}-d^{I}-4(N-2) \hat{k}_{0}^{I}
$$

and note that the fluxes between the blob and ring points are given by:

$$
\Pi_{j(N-1)}^{(I)}=-\frac{1}{4}\left[X^{I}+Q^{-1} d^{I}+4 q_{j}^{-1} k_{j}^{I}\right], \quad \Pi_{j N}^{(I)}=-\frac{1}{4}\left[X^{I}-Q^{-1} d^{I}+4 q_{j}^{-1} k_{j}^{I}\right] .
$$

In particular, the difference of these fluxes is simply the flux through the two-cycle running between the two ring points:

$$
\Pi_{j N}^{(I)}-\Pi_{j(N-1)}^{(I)}=\frac{d^{I}}{2 Q}=\Pi_{(N-1) N}^{(I)} .
$$

For the ring to be far from the black hole, the fluxes $\Pi_{j(N-1)}^{(I)}$ and $\Pi_{j N}^{(I)}$ must be large. For the ring to be thin $\left(\Delta \ll r_{0}\right)$, these fluxes must be of similar order, or $\Pi_{(N-1) N}^{(I)}$ should be small. Hence we are assuming that $\frac{d^{I}}{2 Q}$ is small compared to $X^{I}$. We are also going to want the black hole and the black ring to have similar charges and angular momenta, $J_{R}$, and one of the ways of achieving this is to make $f^{I}, d^{I}$ and $N \hat{k}_{0}^{I}$ of roughly the same order.

Given this, the leading order terms in (4.25) become:

$$
\sum_{j=1}^{N-2} q_{j}\left[\frac{\left(\alpha_{j}-\beta_{j}\right)}{r_{0}}-\alpha_{j} \frac{\Delta}{r_{0}^{2}}\right]=\frac{1}{2} \sum_{I} d^{I} .
$$

One can then determine the ring width, $\Delta$, using (4.19) or (4.20). In particular, when the ring width is small while the ring radius is large, the left-hand side of each of these 
equations is the difference of two very large numbers of similar magnitude. To leading order we may therefore neglect the right-hand sides and use the leading monopole term to obtain:

$$
\beta_{0} \frac{\Delta}{r_{0}} \approx \alpha_{0} \frac{\Delta}{r_{0}}=\left[\sum_{j=1}^{N-2} q_{j} \alpha_{j}\right] \frac{\Delta}{r_{0}} \approx \gamma,
$$

and hence (4.25) becomes:

$$
-\gamma+\sum_{j=1}^{N-2} q_{j}\left(\alpha_{j}-\beta_{j}\right) \approx\left[\frac{1}{2} \sum_{I} d^{I}\right] r_{0} .
$$

Using the explicit expressions for $\alpha_{j}, \beta_{j}$ and $\gamma$, one then finds:

$$
\begin{aligned}
r_{0} \approx\left[4 \sum_{I} d^{I}\right]^{-1}\left[\frac { 1 } { 2 } C _ { I J K } \left(d^{I} f^{J} f^{K}\right.\right. & \left.-f^{I} d^{J} d^{K}\right) \\
& \left.+\left(\frac{3 Q^{2}-4 Q+1}{24 Q^{2}}\right) C_{I J K} d^{I} d^{J} d^{K}-d^{I} \widehat{Q}_{I}\right] .
\end{aligned}
$$

This is exactly the same as the formula for the tube radius that one obtains from (2.35) and (2.34). Note also that we have:

$$
r_{0} \approx\left[4 \sum_{I} d^{I}\right]^{-1}\left[j_{L}-d^{I} \widehat{Q}_{I}\right]
$$

where $j_{L}$ the angular momentum of the supertube (4.11). In making the comparison to the results of [19], recall that for a black ring with a black hole exactly in the center, the embedding radius in the standard, flat $\mathbb{R}^{4}$ metric is given by:

$$
R^{2}=\frac{l_{p}^{6}}{L^{4}}\left[\sum d^{I}\right]^{-1}\left(J_{T}-d^{I} \widehat{Q}_{I}\right)
$$

The transformation between a flat $\mathbb{R}^{4}$ and the GH metric with $V=\frac{1}{r}$ involves setting $r=\frac{1}{4} \rho^{2}$, and this leads to the relation $R^{2}=4 R_{T}$. We therefore have complete consistency with the classical merger result.

Note that the classical merger condition is simply $r_{0} \rightarrow 0$, which is, of course, very natural. This might, at first, seem to fall outside the validity of our approximation, however we will see in the next section that for irreversible mergers one does indeed maintain $\Delta, r_{j} \ll r_{0}$ in the limit $r_{0} \rightarrow 0$. Reversible mergers cannot however be described in this approximation, and have to be analyzed numerically. This is the subject of section 6 . 


\section{Irreversible mergers and scaling solutions}

We now show that an irreversible merger occurs in such a manner that the ring radius, $r_{0}$, the ring width, $\Delta$, and a typical separation of points within the black-hole blob all limit to zero while their ratios all limit to finite values. We will call these scaling solutions, or scaling mergers. As the merger progresses, the throat of the solution becomes deeper and deeper, and corresponding redshift becomes larger and larger. The high-redshift “deep microstates" that result are microstates of a BPS back hole with classically large horizon area.

\subsection{Merging a ring and a black hole}

We use the solution discussed in the previous section, and we decrease the radius of the bubbled ring, $r_{0}$ by decreasing some of its flux parameters. We take all the flux parameters of the $(N-2)$ points in the blob to be parallel:

$$
k_{j}^{I}=\hat{k}_{0}^{I}=k^{I}, \quad j=1, \ldots, N-2,
$$

Further assume that all the GH charges in the black-hole blob obey $q_{j}=(-1)^{j+1}, j=$ $1, \ldots, N-2$. We therefore have

$\widehat{Q}_{I}=2(N-1)(N-3) C_{I J K} k^{J} k^{K}, \quad \widehat{J}_{R}=\frac{8}{3}(N-1)(N-2)(N-3) C_{I J K} k^{I} k^{J} k^{K}$.

Define:

$$
\mu_{i} \equiv \frac{1}{6}\left(N-2-q_{i}\right)^{-1} C_{I J K} \sum_{\substack{j=1 \\ j \neq i}}^{N-2} \Pi_{i j}^{(I)} \Pi_{i j}^{(J)} \Pi_{i j}^{(K)} \frac{q_{j}}{r_{i j}}
$$

then the bubble equations for this blob in isolation (i.e. with no addition bubbles, black holes or rings) are simply:

$$
\mu_{i}=\sum_{I=1}^{3} k^{I}
$$

More generally, in any solution satisfying (5.1), if one finds a blob in which the $\mu_{i}$ are all equal to the same constant, $\mu_{0}$, then the GH points in the blob must all be arranged in the same way as an isolated black hole, but with all the positions scaled by $\mu_{0}^{-1}\left(\sum_{I=1}^{3} k^{I}\right)$. 
Now consider the full set of $N$ points with $\Delta, r_{j} \ll r_{0}$. In the previous sub-section we solved the last two bubble equations and determined $\Delta$ and $r_{0}$ in terms of the flux parameters. The remaining bubble equations are then:

$$
\left(N-2-q_{i}\right) \mu_{i}+\frac{\alpha_{i}}{\left|\vec{r}_{N}-\vec{r}_{i}\right|}-\frac{\beta_{i}}{\left|\vec{r}_{(N-1)}-\vec{r}_{i}\right|}=\sum_{I=1}^{3}\left(\left(N-2-q_{i}\right) k^{I}+\frac{d^{I}}{2}\right)
$$

for $i=1, \ldots, N-2$. Once again we use the multipole expansion in these equations:

$$
\left(N-2-q_{i}\right) \mu_{i}+\frac{\left(\alpha_{i}-\beta_{i}\right)}{r_{0}}-\frac{\alpha_{i} \Delta}{r_{0}^{2}}=\sum_{I=1}^{3}\left(\left(N-2-q_{i}\right) k^{I}+\frac{d^{I}}{2}\right),
$$

It is elementary to show that:

$$
\alpha_{i}-\beta_{i}=\frac{1}{8}\left(j_{L}-d^{I} \widehat{Q}_{I}\right)+\gamma-\frac{1}{8}\left(N-2-q_{i}\right) C_{I J K} d^{I} k^{J} X^{K}
$$

where $X^{I}$ is defined in (4.26). If one now uses this identity, along with (4.30) and (4.33) in (5.6) one obtains:

$$
\begin{aligned}
\left(N-2-q_{i}\right) \mu_{i}-\frac{1}{r_{0}} C_{I J K}\left[\frac{1}{8}\left(N-2-q_{i}\right) d^{I} k^{J} X^{K}\right. & \left.-\left(1-\frac{\alpha_{i}}{\alpha_{0}}\right) \gamma\right] \\
& \approx\left(N-2-q_{i}\right) \sum_{I=1}^{3} k^{I} .
\end{aligned}
$$

Finally, note that:

$$
\alpha_{0}-\alpha_{i}=Q\left(N-2-q_{i}\right) C_{I J K}\left[\frac{1}{32}\left(X^{I}-\frac{1}{Q} d^{I}\right)\left(X^{J}-\frac{1}{Q} d^{J}\right) k^{K}+\frac{1}{6} k^{I} k^{J} k^{K}\right]
$$

which means that all the terms in (5.8) have a factor of $\left(N-2-q_{i}\right)$. Hence the bubble equations (5.5) reduce to:

$$
\begin{aligned}
\mu_{i} \approx\left(\sum_{I=1}^{3} k^{I}\right) & +\frac{1}{r_{0}} C_{I J K}\left[\frac{1}{8} d^{I} k^{J} X^{K}\right. \\
& \left.-\alpha_{0}^{-1} Q \gamma\left(\frac{1}{32}\left(X^{I}-\frac{1}{Q} d^{I}\right)\left(X^{J}-\frac{1}{Q} d^{J}\right) k^{K}+\frac{1}{6} k^{I} k^{J} k^{K}\right)\right] \\
\approx\left(\sum_{I=1}^{3} k^{I}\right) & +\frac{1}{r_{0}} C_{I J K}\left[\frac{1}{8} d^{I} k^{J} X^{K}-\alpha_{0}^{-1} Q \gamma\left(\frac{1}{32} X^{I} X^{J} k^{K}+\frac{1}{6} k^{I} k^{J} k^{K}\right)\right],
\end{aligned}
$$

where we have used the assumption that $X^{I}$ is large compared to $Q^{-1} d^{I}$. 
Observe that the right-hand side of (5.10) is independent of $i$, which means that the first $(N-2) \mathrm{GH}$ points satisfy a scaled version of the equations (5.4) for a isolated, bubbled black hole. Indeed, if $\vec{r}_{i}^{B H}$ are the positions of a set of GH points satisfying (5.4) then we can solve (5.10) by scaling the black hole solution, $\vec{r}_{i}=\lambda^{-1} \vec{r}_{i}^{B H}$, where the scale factor is given by:

$\lambda \approx 1+\frac{1}{r_{0}}\left(\sum_{I=1}^{3} k^{I}\right)^{-1} C_{I J K}\left[\frac{1}{8} d^{I} k^{J} X^{K}-\alpha_{0}^{-1} Q \gamma\left(\frac{1}{32} X^{I} X^{J} k^{K}+\frac{1}{6} k^{I} k^{J} k^{K}\right)\right]$.

Notice that as one approaches the critical "merger" value, at which $\Omega=j_{L}-d^{I} \widehat{Q}_{I}=$ 0 , (5.11) implies that the distance, $r_{0}$, must also scale as $\lambda^{-1}$. Therefore the merger process will typically involve sending $r_{0} \rightarrow 0$ while respecting the assumptions made in our approximations $\left(\Delta, r_{i} \ll r_{0}\right)$. The result will be a "scaling solution" in which all distances in the GH base are vanishing while preserving their relative sizes.

We have indeed verified this picture of the generic merger process by making quite a number of numerical computations, one of which we will present in the next subsection. We tracked one merger through a range where the scale factor, $\lambda$, varied from about 4 to well over 600 . We have also checked that this scaling behavior is not an artefact of axial symmetry. We performed several numerical simulations in which the GH points of the black-hole blob were arranged along a symmetry axis but the bubbled ring approached the black-hole blob at various angles to this axis. We found that the scaling behavior was essentially unmodified by varying the angle of approach.

An important exception to the foregoing analysis arises when the term proportional to $r_{0}^{-1}$ in (5.10) vanishes to leading order. In particular, this happens if we violate one of the assumptions of our analysis, namely, if one has:

$$
X^{I} \equiv 2 f^{I}-d^{I}-4(N-2) k^{I} \approx 0,
$$

to leading order in $Q^{-1} d^{I}$. If $X^{I}$ vanishes one can see that, to leading order, the merger condition is satisfied:

$\Omega \equiv j_{L}-d^{I} \widehat{Q}_{I}=\frac{1}{8} C_{I J K} d^{I}\left[X^{J} X^{K}+8(N-2) k^{J} X^{K}-\frac{1}{3} Q^{-2}(4 Q-1) d^{J} d^{K}+16 k^{J} k^{K}\right] \approx 0$,

and so one must have $r_{0} \rightarrow 0$. However, the foregoing analysis is no longer valid, and so the merger will not necessarily result in a scaling solution. 
An important example of this occurs when $k^{I}, d^{I}$ and $f^{I}$ are all parallel:

$$
k^{I}=k u^{I}, \quad d^{I}=d u^{I}, \quad f^{I}=f u^{I},
$$

for some fixed $u^{I}$. Then the merger condition (5.13) is satisfied to leading order, only when $X \equiv(2 f-d-4(N-2) k)$ vanishes. We will discuss examples of this in the next section, where we will see that the merger process does not involve scaling and the GH points of the ring move very close to the GH points of the black hole.

For non-parallel fluxes it is possible to satisfy the merger condition, (5.13), while keeping $X^{I}$ large, and the result is a scaling solution.

Even if it looks like irreversible mergers progress until the final size on the base vanishes, this is an artifact of working in a classical limit an ignoring the quantization of the fluxes. After taking this into account we can see from (5.10) that $r_{0}$ cannot be taken continuously to zero because the $d^{I}, f^{I}, X^{I}$ and $k^{I}$ are integers of half-integers. Hence, the final result of an irreversible merger is a microstate of a high, but finite, redshift and whose throat only becomes infinite in the classical limit.

In order to find the maximum depth of the throat, one has to find the smallest allowed value for the size of the ensemble of GH points. During the irreversible merger all the distances scale, the size of the ensemble of points will be approximately equal to the distance between the ring blob and the black hole blob, which is given by (4.33). Since $j_{L}-d^{I} \widehat{Q}_{I}$ is quantized, the minimal size of the ensemble of GH points is given by:

$$
\left.r\right|_{\min } \approx \frac{1}{d^{1}+d^{2}+d^{3}} .
$$

More generically, in the scaling limit, the GH size of a solution with left-moving angular momentum $J_{L}$ is

$$
\left.r\right|_{\min } \approx \frac{J_{L}}{d^{1}+d^{2}+d^{3}} .
$$

Since the $d^{I}$ scale like the square-roots of the ring charges, we can see that in the classical limit, $\left.r\right|_{\min }$ becomes zero and the throat becomes infinite.

\subsection{Numerical results for a simple merger}

Given that most of the numerical investigations and most of the derivations we have discussed above use black hole microstate made from a very large number of points, it is quite hard to illustrate explicitly the details of a microstate merger. 
To do this, we investigate a black hole microstate that is made from three points, of GH charges $-n, 2 n+1$, and $-n$, and its merger with a black ring microstate of GH charges $-Q$ and $+Q$. This black hole microstate can be obtained by redistributing the position of the $\mathrm{GH}$ points inside the $\mathrm{BH}$ blob considered in the previous subsection, putting all the +1 charges together and putting half of the -1 charges together on one side of the positive center and the other half on the other side 0

We consider a configuration with five GH centers of charges

$$
q_{1}=-12, \quad q_{2}=25, \quad q_{3}=-12, \quad q_{4}=-20, \quad q_{5}=20 .
$$

The first three points give the black hole "blob," which can be thought as coming from a blob of $N-2=49$ points upon redistributing the GH points as described above. The $k^{I}$ parameters of the black hole points are:

$$
k_{1}^{I}=\left|q_{1}\right| \hat{k}_{0}^{I}, \quad k_{2}^{I}=\left|q_{2}\right| \hat{k}_{0}^{I}, \quad k_{3}^{I}=\left|q_{3}\right| \hat{k}_{0}^{I},
$$

where $\hat{k}_{0}^{I}$ is the average of the $k^{I}$ over the $\mathrm{BH}$ points, defined in (4.1). To merge the ring and the black hole microstates we varied $\hat{k}_{0}^{2}$ while keeping $\hat{k}_{0}^{1}$ and $\hat{k}_{0}^{3}$ fixed:

$$
\hat{k}_{0}^{1}=\frac{5}{2}, \quad \hat{k}_{0}^{3}=\frac{1}{3},
$$

We also fixed the ring parameters $f^{I}$ and $d^{I}$ as follows:

$$
d^{1}=100, \quad d^{2}=130, \quad d^{3}=80, \quad f^{1}=f^{2}=160, \quad f^{3}=350
$$

The relation between these parameters and the $k^{I}$ of the ring is given in (4.3), where $N-2$ (the sum of $\left|q_{i}\right|$ for the black hole points) is now $\left|q_{1}\right|+\left|q_{2}\right|+\left|q_{3}\right|=49$.

10 Since the $k$ parameters on the BH points are the same, the bubble equations give no obstruction to moving BH centers of the same GH charge on top of each other. 


\begin{tabular}{|c|c|c|c|c|c|c|c|}
\hline Parameters & $\hat{k}_{0}^{2}$ & $x_{4}-x_{3}$ & $\frac{x_{4}-x_{3}}{x_{2}-x_{1}}$ & $\frac{x_{2}-x_{1}}{x_{3}-x_{2}}$ & $\frac{x_{2}-x_{1}}{x_{5}-x_{4}}$ & $J_{L}$ & $\frac{Q_{1} Q_{2} Q_{3}-J_{R}^{2} / 4}{Q_{1} Q_{2} Q_{3}}$ \\
\hline 1 & 3.0833 & 175.5 & 2225 & 1.001 & 2.987 & 215983 & .275 \\
\hline 2 & 3.1667 & 23.8 & 2069 & 1.001 & 3.215 & 29316 & .278 \\
\hline 3 & 3.175 & 8.65 & 2054 & 1.001 & 3.239 & 10650 & .279 \\
\hline 4 & 3.1775 & 4.10 & 2049 & 1.001 & 3.246 & 5050 & .279 \\
\hline 5 & 3.178 & 3.19 & 2048 & 1.001 & 3.248 & 3930 & .279 \\
\hline 6 & 3.17833 & 2.59 & 2048 & 1.001 & 3.249 & 3183 & .279 \\
\hline 7 & 3.17867 & 1.98 & 2047 & 1.001 & 3.250 & 2437 & .279 \\
\hline 8 & 3.1795 & .463 & 2046 & 1.001 & 3.252 & 570 & .279 \\
\hline 9 & 3.17967 & .160 & 2045 & 1.001 & 3.253 & 197 & .279 \\
\hline
\end{tabular}

Table 1: Distances between points in the scaling regime. The value of $\hat{k}_{0}^{2}$ is varied to produce the merger, and the other parameters of the configuration are kept fixed: $Q=20, q_{1}=q_{3}=$ $-12, q_{2}=25, \hat{k}_{0}^{1}=\frac{5}{2}, \hat{k}_{0}^{3}=\frac{1}{3}, d^{1}=100, d^{2}=130, d^{3}=80, f^{1}=f^{2}=160, f^{3}=350$. Both the charges and $J_{R}$ remain approximately the same, with $J_{R} \approx 3.53 \times 10^{7}$.

The charges and $J_{R}$ angular momentum of the solutions are approximately

$$
Q_{1} \approx 68.4 \times 10^{3}, Q_{2} \approx 55.8 \times 10^{3}, Q_{3} \approx 112.8 \times 10^{3}, J_{R} \approx 3.53 \times 10^{7}
$$

while $J_{L}$ goes to zero as the solution becomes deeper and deeper. The result of the merger is a microstate of a BMPV black hole that is $28 \%$ below maximal rotation (see the last column of Table 1.)

Numerically solving the bubble equations (2.18), one obtains the positions $x_{i}$ of the five points as a function of $\hat{k}_{0}^{2}$. Some of the results are shown in Table 1. As one can see from the table, a very small increase in the value of $\hat{k}_{0}^{2}$ causes a huge change in the positions of the points on the base. If we were merging classical black holes and classical black rings, this increase would correspond to the black hole and the black ring merging. For microstates, this results in the scaling described above: all the distances on the base become smaller, but their ratios remain fixed.

Analytically verifying that these solutions have no closed timelike curves is not that straightforward, since the quantities in (2.19) have several hundred terms. However, we have investigated numerically and graphically for the possible presence of closed timelike curves, and have found that the equations (2.19) are satisfied throughout the scaling solutions discussed in this sub-section. 


\subsection{A pincer movement: Two rings and a black hole}

The scaling solutions that we have constructed so far do not have $J_{L}=0$, but only achieve this in the extreme limit in which $r_{0} \rightarrow 0$. Thus these solutions have the charges of a black hole of non-zero entropy only in the large-throat limit. While this feature of deep microstates makes them similar to typical microstates, it is interesting to explore whether solutions with $J_{L}=0$ and $Q_{1} Q_{2} Q_{3}>J_{R}^{2} / 4$ must necessarily have long throats, or whether they can also be shallow.

Constructing solutions with $J_{L}=0$ is very simple: One can put identical ring blob on opposite sides of the black-hole blob. Indeed, one can make $\mathbb{Z}_{2}$ invariant solutions in this manner and, as we remarked earlier, this guarantees $J_{L}=0$. From the perspective of the asymptotically $\mathbb{R}^{4}$ base, a ring blob located on axis to the left of the black-hole blob describes a lack ring in one $\mathbb{R}^{2}$ plane in $\mathbb{R}^{4}$, while a ring blob located on axis to the right of the black-hole blob describes a ring in the orthogonal $\mathbb{R}^{2}$ plane. We are thus adding two identical, perpendicular rings to the black hole, thereby guaranteeing $J_{1}=J_{2}$.

We will again consider the merger of these rings with the black hole, and show that having two rings instead of one modifies the analysis above very little. We can again find a scaling behavior, but now $J_{L}=0$ and $Q_{1} Q_{2} Q_{3}>J_{R}^{2} / 4$ throughout the merger. Hence, this microstate will have the charges of a black hole of non-zero entropy when it is shallow (before the scaling regime), when it is deep, and in the intermediate regime.

Once again we consider a system of $N$ GH points but now the first pair and last pair are "exceptional," and correspond to rings. The black-hole blob consists of the $(N-4)$ points in the middle:

$$
\begin{gathered}
q_{1}=Q_{1}, \quad q_{2}=-Q_{1}, \quad q_{N-1}=-Q_{2}, \quad q_{N}=Q_{2}, \quad q_{j}=(-1)^{j+1}, \quad j=3, \ldots, N-2, \\
k_{i}^{I}=k^{I}, \quad j=3, \ldots, N-2 .
\end{gathered}
$$

As before, it is convenient to define 11 :

$$
\begin{aligned}
& d_{1}^{I} \equiv 2\left(k_{1}^{I}+k_{2}^{I}\right), \quad f_{1}^{I} \equiv 2(N-4) k^{I}+\left(1+\frac{1}{Q_{1}}\right) k_{2}^{I}+\left(1-\frac{1}{Q_{1}}\right) k_{1}^{I} \\
& d_{2}^{I} \equiv 2\left(k_{N-1}^{I}+k_{N}^{I}\right), \quad f_{2}^{I} \equiv 2(N-4) k^{I}+\left(1+\frac{1}{Q_{2}}\right) k_{N-1}^{I}+\left(1-\frac{1}{Q_{2}}\right) k_{N}^{I} .
\end{aligned}
$$

11 These definitions are not naturally orthogonal fluxes in cohomology, but they have the virtue of being symmetric between the rings. 
We now have

$\widehat{Q}_{I}=2(N-3)(N-5) C_{I J K} k^{J} k^{K}, \quad \widehat{J}_{R}=\frac{8}{3}(N-3)(N-4)(N-5) C_{I J K} k^{I} k^{J} k^{K}$,

and we define

$$
\begin{gathered}
Q_{I}^{R i n g, i} \equiv C_{I J K} d_{i}^{J} f_{i}^{K} \\
j_{R, i} \equiv \frac{1}{2} C_{I J K}\left(f_{i}^{I} f_{i}^{J} d_{i}^{K}+f_{i}^{I} d_{i}^{J} d_{i}^{K}\right)-\frac{1}{24}\left(1-Q_{i}^{-2}\right) C_{I J K} d_{i}^{I} d_{i}^{J} d_{i}^{K} .
\end{gathered}
$$

Then we find:

$$
Q_{i}=\widehat{Q}_{I}+Q_{I}^{R i n g, 1}+Q_{I}^{R i n g, 2}+C_{I J K} d_{1}^{J} d_{2}^{K}
$$

and

$$
\begin{aligned}
J_{R}=\widehat{J}_{R}+j_{R, 1}+j_{R, 2} & +\left(d_{1}^{I}+d_{2}^{I}\right) \widehat{Q}_{I}+d_{1}^{I} Q_{I}^{\text {Ring }, 2}+d_{2}^{I} Q_{I}^{\text {Ring }, 1} \\
& +\frac{1}{2} C_{I J K}\left(d_{1}^{I} d_{2}^{J} d_{2}^{K}+d_{2}^{I} d_{1}^{J} d_{1}^{K}\right)
\end{aligned}
$$

These formulas agree identically to the formulas that give the charges and angular momenta of two concentric black rings with a black hole in the middle [7]. One can also obtain similar expressions for $J_{L}$, but here we will focus on $\mathbb{Z}_{2}$-invariant solutions and so $J_{L}=0$.

Now set

$$
d_{1}^{I}=d_{2}^{I}=d^{I}, \quad f_{1}^{I}=f_{2}^{I}=f^{I}
$$

and impose $\mathbb{Z}_{2}$ symmetry in the distribution of $\mathrm{GH}$ points. In particular, take the origin to be the center of the distribution and define:

$$
r_{0} \equiv\left|\vec{r}_{N-1}\right|=\left|\vec{r}_{2}\right|, \quad \Delta \equiv\left|\vec{r}_{N}-\vec{r}_{N-1}\right|=\left|\vec{r}_{1}-\vec{r}_{2}\right|
$$

The analysis for $\Delta, r_{i} \ll r_{0}$ proceeds in an almost identical manner to the asymmetric solution described above, but with three modifications:

(i) One shifts $N \rightarrow N-2$ throughout because one now has $N-4$ points, as opposed to $N-2$ points, in the black-hole blob.

(ii) The value of $r_{0}$ is still given by (4.32) and the merger condition is still given by (4.14), but this is no longer related to $J_{L}$ since the latter is always zero.

(iii) While the expression for $r_{0}$ is unchanged, the black-hole blob now feels twice the compression because there are now two rings. Thus (5.10) becomes:

$$
\mu_{i} \approx\left(\sum_{I=1}^{3} k^{I}\right)+\frac{2}{r_{0}} C_{I J K}\left[\frac{1}{8} d^{I} k^{J} X^{K}-\alpha_{0}^{-1} Q \gamma\left(\frac{1}{32} X^{I} X^{J} k^{K}+\frac{1}{6} k^{I} k^{J} k^{K}\right)\right] .
$$


Thus to leading order, the two rings do not influence each other and each settles down as if the other were not there. However the black hole shrinks to about half its previous size. From numerical simulations, we see some amusing features at sub-leading order. In particular, when a single ring is present, the distribution of GH points in the black-hole blob develops a small dipolar asymmetry and the scale factor varies very slightly across the blob (to first sub-leading order). When two rings are present, the symmetry is restored and the variation in the distribution of GH points only appears in the quadrupole moments and the scale factor has only tiny variations across the blob (i.e. to second sub-leading order).

\subsection{The metric structure of the deep microstates}

The physical metric is given by (2.1) and the physical lengths on the three-dimensional base of the $\mathrm{GH}$ space are therefore determined by:

$$
d s_{3}^{3}=\left(Z_{1} Z_{2} Z_{3}\right)^{1 / 3} V d \vec{y} \cdot d \vec{y}
$$

The physical lengths are thus determined by the functions, $Z_{I} V$, and if one has:

$$
\left(Z_{1} Z_{2} Z_{3}\right)^{1 / 3} V \sim \frac{1}{r^{2}}
$$

then the solution looks is an $A d S_{2} \times S^{3}$ black hole throat. In the region where the constants in the harmonic functions become important, this throat turns into an asymptotically flat $\mathbb{R}^{(4,1)}$ region. Near the GH centers that give the black hole bubbles the function $Z_{1} Z_{2} Z_{3}$ becomes constant. This corresponds to the black-hole throat "capping off". As the GH points get closer in the base, the region where (5.34) is valid becomes larger, and hence the throat becomes longer.

As one may intuitively expect, in a scaling solution the ring is always in the throat

of the black hole. Indeed, the term "1" on the right hand side of (5.11) originates from the constant terms in $L_{I}$ and $M$, defined in (2.10). In the scaling regime this term is subleading, which implies the ring is in a region where the 1 in the $L_{I}$ (and hence the $Z_{I}$ ) is also subleading. Hence, the ring lies in the $A d S$ throat of the black-hole blob.

Increasing the scale factor, $\lambda$, in (5.11) means that the bubbles localize in a smaller and smaller region of the GH base, which means that the throat is getting longer and longer. The physical circumference of the throat is fixed by the charges and the angular momentum, and remains finite even though the blob is shrinking on the GH base. Throughout the 
scaling the throat becomes deeper and deeper; the ring remains in the throat, and also descends deeper and deeper into it, in direct proportion to the overall depth of the throat.

On a more mechanistic level, the physical distance through the blob and the physical distance from the blob to the ring are controlled by integrals of the form:

$$
\int\left(Z_{1} Z_{2} Z_{3} V^{3}\right)^{1 / 6} d \ell .
$$

In the throat the behavior of this function is given by (5.34) and this integral is logarithmically divergent as $r \rightarrow 0$. However, the $Z_{I}$ limit to finite values at $\vec{r}=\vec{r}_{j}$ and between two very close, neighboring GH points in the blob, the integral has a dominant contribution of the form

$$
C_{0} \int\left|\left(x-x_{i}\right)\left(x-x_{j}\right)\right|^{-1 / 2} d x
$$

for some constant, $C_{0}$, determined by the flux parameters. This integral is finite and indeed is equal to $C_{0} \pi$. Thus we see that the throat gets very long but then caps off with bubbles of finite physical size.

Since the ring carries charge, its presence will cause the limiting value of the throat size to jump. Figure 4 shows a representative numerical example. These are plots showing $\log \left(Z_{I} V\right)$ against $\log (r)$. In the Coulomb region, away from the ring and the blob, one has

$$
Z_{I} V \sim \frac{Q_{I}}{r^{2}}
$$

where $Q_{I}$ is the charge of the configuration inside a radius $r$. Therefore the log-log plot will show such regions as straight lines of gradient -2 with an intercept fixed by $\log \left(Q_{I}\right)$. Both graphs in Figure 4 show a transition between two such lines, and the "jump" between the lines is accounted for by the jump in the charge as one encounters the ring. The first graph shows the behavior of $Z_{I} V$ taken right through the GH points of the ring, while the second graph shows the behavior $Z_{I} V$ in an orthogonal direction in which one does not pass near the GH points of the ring. As expected, in both instances, the throat widens as one passes the ring radius. 

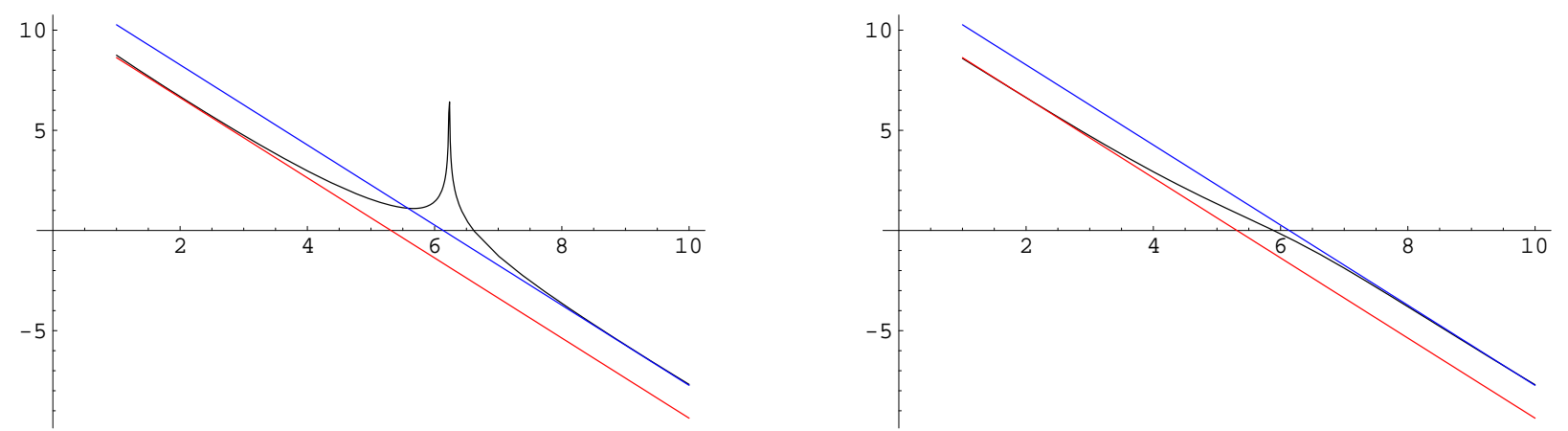

Fig. 4: Typical plots of $\log \left(Z_{I} V\right)$ against $\log (r)$. The straight lines depict exact Coulomb behavior for $Z_{I}$ while the actual solutions transition between two such lines as the ring charge is included. The first graph passes directly through the ring points, while the second goes in an orthogonal radial direction.

\section{Reversible Mergers}

\subsection{Some numerical results in the merger transition}

Once again we performed many numerical simulations for an odd number, $N$, of colinear GH charges. As before, we focused on a system with GH charges $q_{j}=(-1)^{j+1}$, $j=1, \ldots, N-2$ and $q_{N-1}=-Q, q_{N}=Q$. We only considered solutions in which the charges alternate in sign as one goes along the distribution. Before we discuss the results we first need a better geometric understanding of this configuration.

We will take $\left(y_{1}, y_{2}, y_{3}\right)=(x, y, z)$ and suppose that the line of charges lies along the $z$-axis, with the $j^{\text {th }}$ point at $z=z_{j}$. We consider solutions with $z_{j+1}>z_{j}$ so that the charges alternate in sign. We will also denote the polar angle in the $(x, y)$ plane by

$\phi$. One should recall that the GH metric requires one to solve for $\vec{A}$ in (2.3) and for the configuration we are considering we can take

$$
\vec{A} \cdot d \vec{y}=\sum_{J=1}^{N} q_{j} \frac{\left(z-z_{j}\right)}{r_{j}} d \phi
$$

On the $z$-axis, $r_{j}=\left|z-z_{j}\right|$, and since the charges alternate one has, on the $z$-axis:

$$
\begin{aligned}
& \vec{A} \cdot d \vec{y}=(-1)^{\ell} d \phi, \quad \text { for } \quad z_{\ell-1}<z<z_{\ell}, \quad \ell \neq N \\
& \vec{A} \cdot d \vec{y}=-(2 Q-1) d \phi, \quad \text { for } \quad z_{N-1}<z<z_{N},
\end{aligned}
$$

where, for the purposes of the inequality, $z_{0}=-\infty, z_{N+1}=+\infty$. 
Now recall that the change of variables at infinity that takes the GH metric with $V \sim \frac{1}{r}$ to flat $\mathbb{R}^{4}=\mathbb{R}^{2} \times \mathbb{R}^{2}$ with coordinates $\left(u, \theta_{1}\right)$ and $\left(v, \theta_{2}\right)$ in each of the $\mathbb{R}^{2}$ planes involves taking:

$$
\theta_{1}=\frac{1}{2}(\psi+\phi), \quad \theta_{2}=\frac{1}{2}(\psi-\phi) .
$$

This, combined with (6.2), means that the GH fiber defined by $(d \psi+A)$ alternates between being in the $\left(u, \theta_{1}\right)$ plane and the $\left(v, \theta_{2}\right)$ plane. In particular, if the two exceptional points are far away, then there is a long interval $\left(z_{N-2}<z<z_{N-1}\right)$ with GH fiber in the $\left(u, \theta_{1}\right)$ plane. Once one pushes this through the complete change of variables, one finds that, at large scales, such a ring blob resembles a supertube in the $\left(u, \theta_{1}\right)$ plane. More generally, whether a blob like this corresponds to a ring in the $\left(u, \theta_{1}\right)$ plane or the $\left(v, \theta_{2}\right)$ plane depends upon whether the large interval on the $z$-axis has $(d \psi+A)$ equal to $(d \psi+d \phi)$ or $(d \psi-d \phi)$, respectively.

In the simulations we set all the $k_{j}^{I}=+1$ in the initial blob $(j=1, \ldots, N-2)$, and took $d^{I}=d$ and $f^{I}=f$, but otherwise arbitrary. We took $Q$ to be fairly large (usually about 30). We then did many series of simulations in which we adjusted $d$ but kept the ratio, $f / d$, fixed. The ring width, $\Delta$, becomes smaller when the values of $f / d$ get larger. The value of $d$ was adjusted so as to move the two exceptional "black ring" points in from a great distance from the black-hole blob. The numerical solutions tend to be very unstable, or rather delicate, particularly when the black ring points are near the bubbling black hole. This is because there are a great many solutions to the bubble equations in which points are re-ordered. These solutions are very "close to one another" when bubbles become small, and the numerical algorithms readily jump between different branches of the solution space. In spite of this, if one carefully adjusts the solution adiabatically and uses the previous solution as initial data to find the next solution, one can follow a single branch in the solution space.

At merger (defined by $J_{1}=J_{2}$ ) we found that the assumption that the two ring points are at a distance from the blob that is of the same order as the size of $r_{0}$ in (4.33) is no longer valid. It turns out that as one comes close to satisfying the merger condition, $r_{0}$ is still somewhat larger than $r_{i}$, but $\Delta / r_{0}$ is extremely small. Dropping the $\Delta / r_{0}$ term in the derivation of the estimate of $r_{0}$ means that when the merger condition $\left(J_{1}=J_{2}\right)$ is satisfied, one still has:

$$
r_{0} \approx Q^{-1}\left[24 \sum_{I} d^{I}\right]^{-1} C_{I J K} d^{I} d^{J} d^{K} .
$$


While we have not been very careful in the derivation of this estimate, it turns out to work rather well in the numerical simulations of mergers with parallel fluxes. For example, for the result depicted in the first graph of Figure 5, we found that the exceptional points were 37.95 units (taking $k=1$ ) from the center of the blob. The formula, (6.4), gives $r_{0} \approx 36.68$. We also found that at the merger value one could bring the exceptional points arbitrarily close to the edge of the blob by taking $Q$ to be large enough. This is also consistent with (6.4),

In all the numerical simulations, the blob maintained the BMPV profile of Figure 3 so long as the exceptional points remained relatively far away. However, as the exceptional points approach the blob closely, the blob undergoes a "phase transition." If the merger value of $r_{0}$ is still significantly larger than the blob size (as it is for the results depicted in Figure 5) then bringing the exceptional points near the blob entails tracking the solution past the merger condition. That is, one follows the solution as $J_{1}-J_{2}$ passes from positive to negative values. We found that the two exceptional points generically do not enter the blob (at least on this branch of solution space) but get very close to it, and actually "steal" the outermost GH center and form a cluster of three points of net GH charge +1 . This cluster then moves away back towards the original distant position of the exceptional points. Throughout this process $J_{1}-J_{2}$ monotonically decreases.

The "theft" of a +1 GH point renders the blob neutral, and makes it to change from a black-hole blob to a ring blob, with the concomitant redistribution of dipole pairings. This "unzipping" phase transition in the blob happens extremely quickly as one varies $d$ below the merger point. Typical results are depicted in Figure 5.

Before the transition the "large interval" lies between $z_{N-2}$ and $z_{N-1}$, and so the black ring points correspond to a ring in the $\left(u, \theta_{1}\right)$ plane. After the transition, as the cluster of three charges moves away, the "large interval" now lies between $z_{N-3}$ and $z_{N-2}$. Therefore, at large scales the resulting configuration corresponds to a black hole microstate surrounded by a black ring microstate in the $\left(v, \theta_{2}\right)$ plane! Thus we are exploring a branch of the solution space in which a bubbling supertube in one plane collapses into an extremal black hole and a then a supertube emerges from the extremal black hole, but now in the orthogonal plane. All the while, $J_{1}-J_{2}$ decreases. These obviously all represent reversible mergers.

There are evidently a vast number of solutions and probably a vast number of branches in the moduli space of locations of GH points. The branch we explored was probably the 

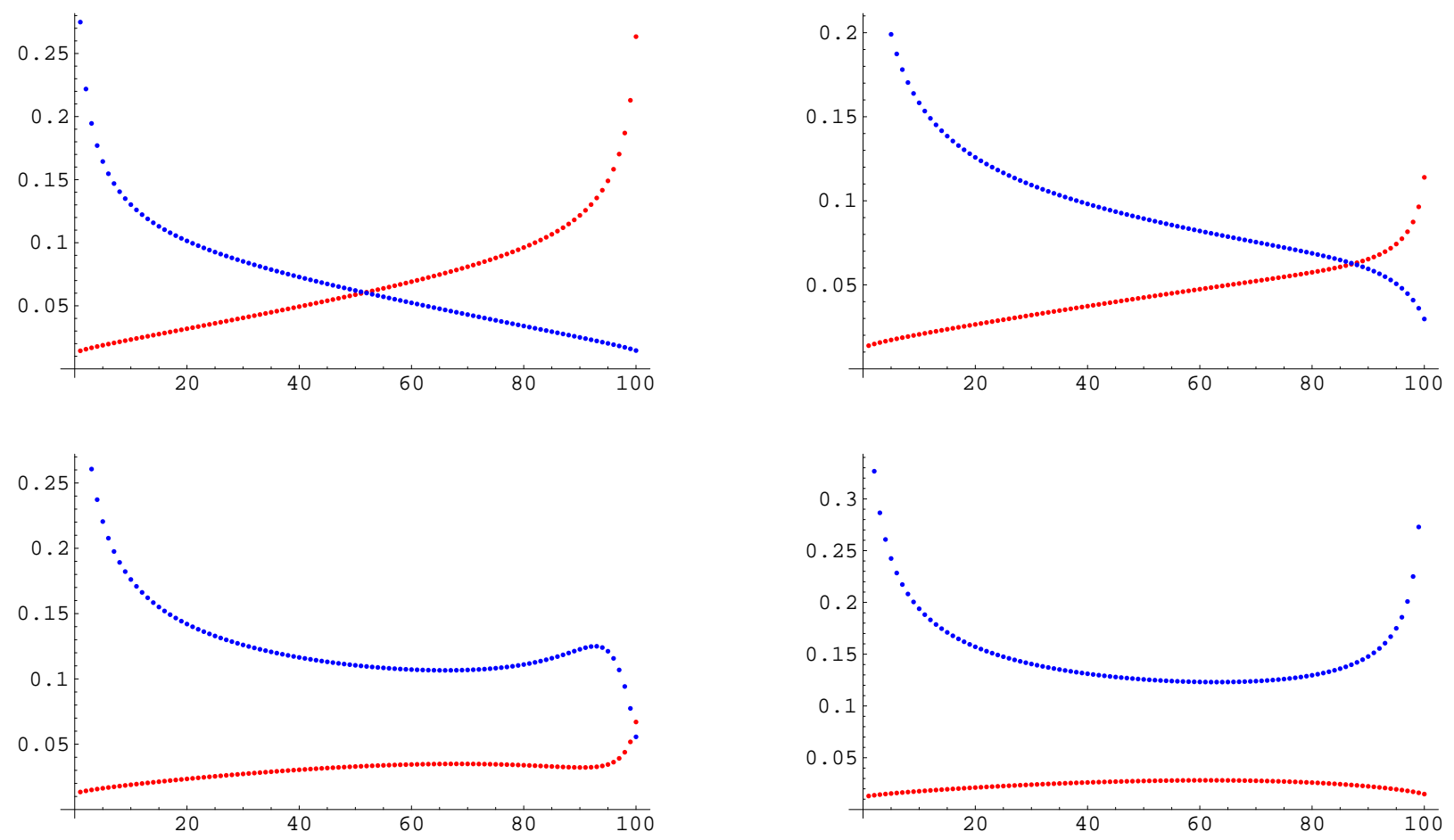

Fig. 5: These graphs show the distances between neighboring GH points for $N=203$, $Q=30, f=4 d$, but with $d$ decreasing from its merger value, $d=114.9074$, in the first graph to $d=114.84$ in the top-right, $d=114.825$ in the bottom-left and $d=114.80$ in the bottom right. Note how the distributions change between that of a black-hole blob to that of a ring blob as $d$ changes only by $0.1 \%$. In the first graph the blob diameter is 14.02 while the space to the first exceptional point is $\left|z_{N-2}-z_{N-1}\right|=30.94$. In the second graph these quantities are 14.46 and 0.999, in the third they are 16.02 and 0.547. In the fourth graph, the "theft" has happened: The blob of $N-3$ points has radius 17.55 and the "large distance" is now $\left|z_{N-3}-z_{N-2}\right|=4.908$.

simplest that allowed $J_{1}-J_{2}$ to decrease smoothly while preserving the order and colinearity of the GH points. There are almost certainly many other solutions in which the GH points are reordered, perhaps with the exceptional points penetrating more deeply into the blob. There are also quite probably solutions in which more GH points are stolen from the blob.

\subsection{Estimating the size of shallow microstates from mergers}

As we have seen is section 5, deep microstates have very long black-hole-like throats, and it is clear that they have the same size and macroscopic features as the black hole. However, it is interesting to explore whether "shallow" microstates, that do not have long 
throats, also have the same macroscopic properties as the actual black hole. A good way to estimate this size is to probe the shallow microstates using mergers.

The irreversible mergers that we considered in section 5 can, by no means, be considered "probing". The merger collapses the foam, resulting in a deep microstate. This is partially because the "probing ring" had comparable charges to those of the black hole. To find the "size" of a shallow microstate one needs to merge it with a small probe-like ring microstate and perform as nearly a reversible merger as possible. The calculations of the previous sub-section describe exactly such a process.

As we have seen, the reversible merger happens when the ring foam sits in the vicinity of the black hole foam. Therefore, at the merger point the shallow microstates essentially touch, resulting in another shallow microstate. In the merger of classical black rings and classical black holes, the horizons of the two objects also touch at the merger point. Hence, reaching the horizon of the black hole corresponds in the microstate picture to reaching the "edge" of the shallow microstates. This indicates that shallow microstate geometries also have the same size and macroscopical properties as their corresponding classical black holes.

\section{Deep Microstates and Typical Microstates}

As we have seen in the previous section, the throats of the deep microstates become infinite in the classical limit. Nevertheless, taking into account flux quantization one can find that the $\mathrm{GH}$ radius of microstates does not go all the way to zero, but to a finite value (5.15), which corresponds to setting $J_{L}=1$.

One can estimate the energy gap of the solution by considering the lightest possible state at the bottom of the throat, and estimating its energy as seen from infinity. The lightest massive particle one can put on the bottom of the throat is not a Planck-mass object, but a Kaluza-Klein mode on the $S^{3}$. Its mass is

$$
m_{K K}=\frac{1}{R_{S^{3}}}=\frac{1}{\left(Q_{1} Q_{2} Q_{3}\right)^{\frac{1}{6}}}
$$

and therefore the mass gap in a microstate of size $r_{\min }$ in the GH base is:

$$
\Delta E_{r_{0}}=\left.m_{K K} \sqrt{g_{00}}\right|_{r=r_{\min }}=\left.m_{K K}\left(Z_{1} Z_{2} Z_{3}\right)^{-1 / 3}\right|_{r=r_{\min }}=\frac{r_{\min }}{\left(Q_{1} Q_{5} Q_{P}\right)^{1 / 2}}
$$


Since in the case of a ring-hole merger $r_{\min }$ depends on the sum of the $d^{I}$, its relation with the total charges of the system is not straightforward. Nevertheless, we can consider a regime where $Q_{1} \sim Q_{5}>Q_{P}$, and in this regime the dipole charge that dominates the sum in (5.16) is $d^{3} \approx \sqrt{\frac{Q_{1} Q_{5}}{Q_{P}}}$. Hence

$$
r_{\min }=\frac{J_{L}}{d^{3}} \approx J_{L} \sqrt{\frac{Q_{P}}{Q_{1} Q_{5}}},
$$

giving

$$
\Delta E_{r_{0}} \approx \frac{J_{L}}{Q_{1} Q_{5}} .
$$

For $J_{L}=1$ this matches the charge dependence of the mass gap of the black hole [29].

This M-theory frame calculation is done in the limit $Q_{1} \sim Q_{5}>Q_{P}$, which is the limit in which the solution, when put into the D1-D5-P duality frame, becomes asymptotically $A d S_{3} \times S^{3} \times T^{4}$. 2 Hence, the mass gap computed in the bulk (7.4), should match the mass gap of the dual microstate in the D1-D5 CFT.

As it is well known (see [31,32] for reviews) the states of this CFT can be characterized by various ways of breaking an effective string of length $N_{1} N_{5}$ into component strings. BPS momentum modes on these component strings carry $J_{R}$. The fermion zero modes of each component allow it in addition to carry one unit of $J_{L}$. The typical CFT microstates that contribute to the entropy of the three-charge black hole have one component string [21]; microstates dual to objects that have a macroscopically large $J_{L}$ have the effective string broken into many component strings [33, 33, 30]. Hence, the only way a system can have a large amount $J_{L}$ is to be have many component strings. The CFT mass gap corresponds to exciting the longest component string, and is proportional to the inverse of its length.

The formula (7.4) immediately suggests a dual for the deep microstates. Consider a long effective string of length $N_{1} N_{5}$ broken into $J_{L}$ component strings of equal length. Each component string can carry one unit of left-moving angular momentum, totaling up to $J_{L}$. The length of each component string is

$$
l_{\text {component }}=\frac{N_{1} N_{5}}{J_{L}}
$$

and hence the CFT mass gap is

$$
\Delta E_{C F T} \approx \frac{J_{L}}{N_{1} N_{5}}
$$

12 As shown in [30], in this limit $d^{1}+d^{2}+d^{3} \rightarrow d^{3}$, which justifies going from (5.16) to (7.3). 
This agrees with both the $J_{L}$ dependence and the charge dependence of the bulk mass gap. While we have been cavalier about various numerical factors of order one, the agreement that we have found suggests that deep microstates of angular momentum $J_{L}$ are dual to CFT states with $J_{L}$ component strings. If this is true, then the deepest microstates, which have $J_{L}=1$, correspond to states that have only one component string, of length $N_{1} N_{5}$. This is a feature that typical microstates of the three-charge black hole have, and the fact that deep microstates share this feature is quite remarkable.

Our analysis here has been rather heuristic. It would be very interesting to examine this issue in greater depth by finding, at least approximate solutions to the wave equation in these backgrounds, or performing a time-of-flight analysis along the lines of [33, 34, 15.

\section{Final Remarks}

We have examined bubbled solutions constructed using a generalized GibbonsHawking base space. We have shown that if the GH points are localized in blobs of total GH charge +1 , then the solutions correspond to microstates of the maximally-spinning BPS black hole. Similarly, blobs whose total GH charge is zero correspond to zero-entropy black ring microstates.

We found numerically that inside a bubbling black ring the GH centers form tight neutral dipole pairs, separated from each other by relatively large distances. On the other hand, inside the black hole, the dipoles are only tightly bound around the edges, and in the middle one finds an equally spaced distribution of positive and negative GH charges.

We have then investigated the merger of bubbling black rings and bubbling black holes, and have found two types of mergers. If the final state has the charges corresponding to a black hole of classically large horizon area (this would correspond to an irreversible merger of a black hole and a black ring), then during the merger the distances between the GH points on the base shrink, while the ratios of all these distances remain fixed. The physical size of the bubbles that form the solution remains the same, but the throat of the solutions becomes deeper and deeper. We have therefore called these solutions "deep" microstates.

Classically this scaling goes on forever, until all the distances in the base are zero. However, the fact that the dipole charges are quantized does not allow one to take them exactly to the value which would correspond to an infinite throat; hence, the depth of the microstate is very large but finite for finite charges, and only becomes infinite in the classical limit. 
When the length of the throat of a deep microstate is very large, this solution more and more accurately resembles a genuine BPS back hole (which has an infinite throat), except for the presence of a horizon. This feature of the deep microstates makes them very attractive candidates for being typical $\mathrm{BH}$ microstates.

The similarity of the deep microstates to the typical CFT microstates can also be observed from the crude $A d S$-CFT analysis we have attempted in Section 7 . We have argued that deep microstates have the right mass gap to be dual to CFT states described by one long component string, and are thus similar in this respect to the typical microstates. It is quite remarkable that the calculation that relates deep microstates of angular momentum $J_{L}$ to states with $J_{L}$ component strings is independent of the number of GH centers. One can speculate that solutions with different numbers of GH points may be related to CFT states that have different distributions of momentum modes on the component strings. It would be very important to establish whether such a relation exists, and more generally to undertake an in-depth CFT investigation of deep microstates. This will be very fruitful, and will hold the key to relating bubbling microstates to their CFT counterparts.

We have also used the merger of a black hole with two concentric black rings to obtain microstate solutions that correspond to a black hole with classically large entropy but which can range between shallow and deep microstates. Since the redshift of the shallow and intermediate solutions does not diverge in the classical limit, it is likely that they are "less typical" than the deep microstates, or that they are not microstates of the black hole, but microstates of a configuration of a black hole with two concentric black rings. It would be very interesting to explore how generic these solutions are, and what their CFT dual is. It would also be very interesting to try to construct deep black ring microstates (which would be similar to typical microstates of a black ring), and to analyze the merger of two deep microstates, or of a shallow and a deep microstate.

We have also analyzed mergers in which no scaling takes place, and which result in "shallow" microstates. We have found that during these mergers two shallow microstates join, forming a larger shallow microstate. We have used this fact to estimate the size of shallow microstates, and to argue that for the maximally-spinning black hole, the bubbles extend to the location of the would-be classical horizon. This is a strong indication that the shallow microstate geometries also have features that typical $\mathrm{BH}$ microstates should have.

While the Gibbons-Hawking hyper-Kähler geometries are simple and "highly computable," it is likely that to make significant further progress in describing and counting the 
microstates of even ordinary BMPV black holes (with non-maximal angular momentum), we will have to get some deeper understanding of the broader class of four-dimensional generalized hyper-Kähler geometries. An obvious first step is to look at geometries that still possess a $U(1)$ isometry, but one that is not tri-holomorphic. Such geometries are determined by the $S U(\infty)$ Toda equation [35,36]. In particular, we expect this class of solutions to describe more general microstates of black holes and black rings, and perhaps even axially aligned families of such objects. Such geometries will also describe the bubbled versions of supertubes with arbitrary charge densities [37] and perhaps with shape modes that are restricted to a plane. As we remarked in the introduction, such solutions are also needed to describe the merging of microstates of two co-axial BMPV black holes.

Even more generally, supersymmetry merely requires that the base be hyper-Kähler, and so the most general, and presumably by far the most numerous solutions will have no isometries at all. It would be useful to see if twistor methods or generalized Legendre transformations [38,39,40,41] could be adapted to this problem. These methods linearize the Monge-Ampére equation that underlies the general hyper-Kähler metric, but in practice it is hard to obtain explicit metrics from this method. On the other hand, if one is trying to quantize and count such metrics then this may well be the natural way to proceed. Another way to obtain more general families of microstate geometries would be to look for solutions that have a nontrivial metric on the $T^{6}$, and cannot be reduced to five dimensions [34, [17]. There is evidently a considerable number of interesting geometries yet to be discovered and studied, and these geometries lie at the heart of understanding the interior structure of black holes.

\section{Acknowledgments}

We would like to thank Juan Maldacena for interesting discussions. IB would also like to thank the Santa Barbara KITP and the Aspen Center for Physics for hospitality during various stages at this project. The work of NW and CWW is supported in part by the DOE grant DE-FG03-84ER-40168. The work of IB is supported in part by the NSF grants PHY-0503584 and PHY-990794.

\section{Appendix A. More Details on the Entropy of the Foam}

When a foam has a very large number of centers, one can obtain many solutions with

the same charges and angular momenta, but which have different flux parameters. In 
[1] we estimated the entropy of such arrangements (which we referred to as "topological" entropy) and found that it is proportional to $Q^{1 / 4}$. Since all the details of this estimate were not given in [1] we devote this Appendix to this task.

If we work in $\mathrm{M}$ theory on $T^{6}$, then (3.3) implies that a black hole foam has

$$
\sum k_{i}^{1}=\frac{1}{2} \sqrt{\frac{Q_{2} Q_{3}}{Q_{1}}}
$$

and similarly for $\sum k_{i}^{2}$ and $\sum k_{i}^{3}$. Hence, the total charges do not depend on the number, GH charges or individual $k_{i}^{I}$ of the GH points, but only on the sums of the $k_{i}$. In [1] we found that the non-trivial entropy coming simply from the many possibilities of choosing the positive, half-integer, $k_{i}^{1}$ subject to the constraint (A.1) is

$$
S=2 \pi \sqrt{\frac{1}{6}\left(\frac{Q_{2} Q_{3}}{Q_{1}}\right)^{1 / 2}}
$$

Naively there should be similar factors coming from partitioning $k_{i}^{2}$ and $k_{i}^{3}$, which would lead to a "topological" entropy :

$$
S_{\text {topological }}=2 \pi\left(\sqrt{\frac{1}{6}\left(\frac{Q_{2} Q_{3}}{Q_{1}}\right)^{1 / 2}}+\sqrt{\frac{1}{6}\left(\frac{Q_{1} Q_{2}}{Q_{3}}\right)^{1 / 2}}+\sqrt{\frac{1}{6}\left(\frac{Q_{1} Q_{3}}{Q_{2}}\right)^{1 / 2}}\right) .
$$

There are, however some subtleties. First, the partitioning of $k_{i}^{1}, k_{i}^{2}$ and $k_{i}^{3}$ is not completely independent. A bubble will collapse unless all three fluxes are non-zero, and so we should count the ways of having non-zero partitions of all the $k_{i}^{I}$ over $N$ bubbles and then sum over $N$. It is, however, relatively easy to show that this additional constraint only modifies the entropy (A.3) by terms that are logarithmic in the charges.

To see this, it is convenient to define:

$$
g(z, q) \equiv \prod_{n=1}^{\infty} \frac{1}{\left(1-z q^{n}\right)}=\sum_{N=1}^{\infty} d_{N}(z) q^{N}
$$

The coefficient of $z^{m}$ in $d_{N}(z)$ is the number of (non-zero) partitions of $N$ into $m$ positive integers. The counting function we want is:

$$
\begin{aligned}
G\left(w, z ; q_{1}, q_{2}, q_{3}\right) & \equiv g\left(z, q_{1}\right) g\left(w, q_{2}\right) g\left(z^{-1} w^{-1}, q_{3}\right) \\
& =\sum_{N_{1}, N_{2}, N_{3}=1}^{\infty} d_{N_{1} N_{2} N_{3}}(w, z) q_{1}^{N_{1}} q_{2}^{N_{2}} q_{3}^{N_{3}} .
\end{aligned}
$$


To count the entropy one must find the coefficient of $w^{0} z^{0}$ in $d_{N_{1} N_{2} N_{3}}(w, z)$, where $N_{J}=$ $\left(Q_{1} Q_{2} Q_{3}\right)^{1 / 2} / Q_{J}$. Selecting these powers of $w$ and $z$ guarantees that all the partitions are taken over the same number of bubbles. One can perform the asymptotic analysis of this partition function using steepest descent methods 23 . One finds that for large $N_{J}$, one has

$$
d_{N_{1} N_{2} N_{3}}(w, z) \sim \exp \left(2\left(\sqrt{N_{1} \operatorname{Li}_{2}(z)}+\sqrt{N_{2} \operatorname{Li}_{2}(w)}+\sqrt{N_{3} \operatorname{Li}_{2}\left(z^{-1} w^{-1}\right)}\right)\right)
$$

provided that $z, w$ lie in appropriate regions around $z=w=1$. The functions, $\mathrm{Li}_{2}$, are standard dilogarithms. One can extract the coefficient of $w^{0} z^{0}$ by contour integrals, but it is easy to see that the leading exponential behavior of any such integral is dominated by the value of (A.6) at $z=w=1$, and using $\operatorname{Li}_{2}(1)=\frac{\pi^{2}}{6}$, one arrives at (A.3).

There is a simple, intuitive way of arriving at this result. The coefficients, $d_{N}(z)$, in (A.4) are polynomials of degree $N$ in $z$. The value $d_{N}(1)$ grows as $\exp (2 \pi \sqrt{N / 6})$. Thus, a "typical coefficient" in the polynomial, $d_{N}(z)$, grows as $N^{-1} \exp (2 \pi \sqrt{N / 6})$. Therefore, restricting to a single power of $z$ simply leads to $\log (N)$ corrections to the entropy.

The second subtlety is that, given the $k_{j}^{1}$, there are also further constraints on $k_{j}^{2}$ and $k_{j}^{3}$ imposed by the global absence of CTC's. These conditions are somewhat more difficult to handle but we believe that the bubble equations, combined with some suitable positivity conditions on the fluxes, will suffice to guarantee the conditions in (2.19), and hence that (A.3) is correct to leading order. Independent of these subtleties and the constraints on $k_{j}^{2}$ and $k_{j}^{3}$ for a given set of $k_{j}^{1}$, we see from (A.2) alone that the topological entropy grows as $Q^{1 / 4}$.

As we discussed in [1], these calculations indicate that the topological entropy is not enough to account for the entropy of the black hole; to capture the latter one would have to consider microstates that do not reduce to four dimensional multi-center solutions, and that are determined by arbitrary functions [33, 34, 43].

13 See, for example, 42], pp 116-118. 


\section{References}

[1] I. Bena, C. W. Wang and N. P. Warner, "The foaming three-charge black hole," arXiv:hep-th/0604110.

[2] I. Bena and N. P. Warner, "Bubbling supertubes and foaming black holes," arXiv:hepth/0505166.

[3] P. Berglund, E. G. Gimon and T. S. Levi, "Supergravity microstates for BPS black holes and black rings," arXiv:hep-th/0505167.

[4] J. P. Gauntlett, J. B. Gutowski, C. M. Hull, S. Pakis and H. S. Reall, "All supersymmetric solutions of minimal supergravity in five dimensions," Class. Quant. Grav. 20, 4587 (2003) arXiv:hep-th/0209114.

[5] J. B. Gutowski and H. S. Reall, "General supersymmetric AdS(5) black holes," JHEP 0404, 048 (2004) arXiv:hep-th/0401129.

[6] I. Bena and N. P. Warner, "One ring to rule them all ... and in the darkness bind them?," Adv. Theor. Math. Phys. 9 (2006) 1-35 arXiv:hep-th/0408106.

[7] J. P. Gauntlett and J. B. Gutowski, "General concentric black rings," Phys. Rev. D 71, 045002 (2005) arXiv:hep-th/0408122.

[8] I. Bena and P. Kraus, "Three charge supertubes and black hole hair," Phys. Rev. D 70, 046003 (2004) arXiv:hep-th/0402144.

[9] H. Elvang, R. Emparan, D. Mateos and H. S. Reall, "A supersymmetric black ring," Phys. Rev. Lett. 93, 211302 (2004) arXiv:hep-th/0407065.

[10] H. Elvang, R. Emparan, D. Mateos and H. S. Reall, "Supersymmetric black rings and three-charge supertubes," Phys. Rev. D 71, 024033 (2005) arXiv:hep-th/0408120.

[11] S. D. Mathur, "The fuzzball proposal for black holes: An elementary review," Fortsch. Phys. 53, 793 (2005) arXiv:hep-th/0502050.

[12] I. Bena, Lectures given at "Winter School on Attractor Mechanism," INFN-Laboratori Nazionali di Frascati, Italy, March 2006, to appear.

[13] O. Lunin, "Adding momentum to D1-D5 system," JHEP 0404, 054 (2004) arXiv:hepth/0404006].

[14] S. Giusto, S. D. Mathur and A. Saxena, "Dual geometries for a set of 3-charge microstates," Nucl. Phys. B 701, 357 (2004) arXiv:hep-th/0405017.

[15] S. Giusto, S. D. Mathur and A. Saxena, "3-charge geometries and their CFT duals," Nucl. Phys. B 710, 425 (2005) arXiv:hep-th/0406103.

[16] S. Giusto and S. D. Mathur, "Geometry of D1-D5-P bound states," Nucl. Phys. B 729, 203 (2005) arXiv:hep-th/0409067.

[17] S. Giusto, S. D. Mathur and Y. K. Srivastava, "A microstate for the 3-charge black ring," arXiv:hep-th/0601193.

[18] G. W. Gibbons and P. J. Ruback, "The Hidden Symmetries Of Multicenter Metrics," Commun. Math. Phys. 115, 267 (1988). 
[19] I. Bena, C. W. Wang and N. P. Warner, "Sliding rings and spinning holes," arXiv:hepth/0512157.

[20] A. Strominger and C. Vafa, "Microscopic Origin of the Bekenstein-Hawking Entropy," Phys. Lett. B 379, 99 (1996) arXiv:hep-th/9601029].

[21] J. C. Breckenridge, R. C. Myers, A. W. Peet and C. Vafa, "D-branes and spinning black holes," Phys. Lett. B 391, 93 (1997) arXiv:hep-th/9602065.

[22] I. Bena, "Splitting hairs of the three charge black hole," Phys. Rev. D 70, 105018 (2004) arXiv:hep-th/0404073.

[23] J. P. Gauntlett and J. B. Gutowski, "Concentric black rings," Phys. Rev. D 71, 025013 (2005) arXiv:hep-th/0408010.

[24] F. Denef, "Supergravity flows and D-brane stability," JHEP 0008, 050 (2000) arXiv:hep-th/0005049.

[25] B. Bates and F. Denef, "Exact solutions for supersymmetric stationary black hole composites," arXiv:hep-th/0304094.

[26] I. Bena and P. Kraus, "Microstates of the D1-D5-KK system," Phys. Rev. D 72, 025007 (2005) arXiv:hep-th/0503053.

[27] A. Saxena, G. Potvin, S. Giusto and A. W. Peet, "Smooth geometries with four charges in four dimensions," arXiv:hep-th/0509214.

[28] V. Balasubramanian, E. G. Gimon and T. S. Levi, "Four dimensional black hole microstates: From D-branes to spacetime foam," arXiv:hep-th/0606118.

[29] J. M. Maldacena and L. Susskind, "D-branes and Fat Black Holes," Nucl. Phys. B 475, 679 (1996) arXiv:hep-th/9604042.

[30] I. Bena and P. Kraus, "Microscopic description of black rings in AdS/CFT," JHEP 0412, 070 (2004) arXiv:hep-th/0408186.

[31] O. Aharony, S. S. Gubser, J. M. Maldacena, H. Ooguri and Y. Oz, "Large N field theories, string theory and gravity," Phys. Rept. 323, 183 (2000) arXiv:hep-th/9905111.

[32] J. R. David, G. Mandal and S. R. Wadia, "Microscopic formulation of black holes in string theory," Phys. Rept. 369, 549 (2002) arXiv:hep-th/0203048.

[33] O. Lunin and S. D. Mathur, "AdS/CFT duality and the black hole information paradox," Nucl. Phys. B 623, 342 (2002) arXiv:hep-th/0109154.

[34] O. Lunin, J. M. Maldacena and L. Maoz, "Gravity solutions for the D1-D5 system with angular momentum," arXiv:hep-th/0212210.

[35] C. P. Boyer and J. D. . Finley, "Killing Vectors In Selfdual, Euclidean Einstein Spaces," J. Math. Phys. 23, 1126 (1982).

[36] J. Gegenberg and A. Das, "Stationary Riemannian space-times with self-dual curvature," Gen. Rel. Grav. 16 (1984) 817.

[37] I. Bena, C. W. Wang and N. P. Warner, "Black rings with varying charge density," HEP 0603, 015 (2006) arXiv:hep-th/0411072. 
[38] N. J. Hitchin, A. Karlhede, U. Lindstrom and M. Rocek, "Hyperkahler Metrics And Supersymmetry," Commun. Math. Phys. 108, 535 (1987).

[39] A. Karlhede, U. Lindstrom and M. Rocek, "Hyperkahler Manifolds And Nonlinear Supermultiplets," Commun. Math. Phys. 108, 529 (1987).

[40] U. Lindstrom and M. Rocek, "New Hyperkahler Metrics And New Supermultiplets," Commun. Math. Phys. 115, 21 (1988).

[41] I. T. Ivanov and M. Rocek, "Supersymmetric sigma models, twistors, and the AtiyahHitchin metric," Commun. Math. Phys. 182, 291 (1996) arXiv:hep-th/9512075.

[42] M. B. Green, J. H. Schwarz and E. Witten, "Superstring Theory. Vol. 1: Introduction," [43] A. Saxena, to appear. 